\title{
Simulation and high-precision wavelength determination of noisy 2D Fabry-Pérot interferometric rings for direct-detection Doppler lidar and laser spectroscopy
}

\author{
Markus C. Hirschberger and Gerhard Ehret \\ Deutsches Zentrum für Luft- und Raumfahrt (DLR), Institut für Physik der Atmosphäre (IPA), \\ Oberpfaffenhofen, Münchner Str. 20, 82234 Wessling, Germany \\ e-mail: markus.hirschberger@dlr. de, phone: 08153/28-2554, fax: 08153/28-1271
}

Received: 28 July 2010 / Revised version: 13 December 2010

\begin{abstract}
Doppler wind lidar (DWL) measurements by the fringe-imaging technique in front of aircrafts at flight speed require rapid processing of backscattered signals. We discuss the measurement principle to derive the $3 \mathrm{D}$ wind vector from three line-of-sight (LOS) measurements. Then we simulate realistic fringe patterns of a Fabry-Pérot-interferometer (FPI) on a $2 \mathrm{D}$ charge-coupled device (CCD) localized at the focal plane behind it, taking atmospheric and instrument properties like scattering and noise into account. A laser at $355 \mathrm{~nm}$ with pulse energies of $70 \mathrm{~mJ}$ at $100 \mathrm{~Hz}$ repetition rate and a range bin of only $10 \mathrm{~m}$ were assumed. This yields count rates of 24 (13) million photons per pulse at $56(76) \mathrm{m}$ distance and $8.5 \mathrm{~km}$ altitude, that are distributed on a CCD with up to $960 \times 780$ pixels without intensification and therefore generate noisy pixel signals. We present two methods for the precise determination of the radii, i.e. wavelengths, of these simulated FPI rings and show that both are suitable for eliminating pixel noise from the output and coping with fringe broadening by Rayleigh scattering. One of them proves to reach the accuracy necessary for LOS velocity measurements. A standard deviation of $2.5 \frac{\mathrm{m}}{\mathrm{s}}$ including center determination can be achieved with only 20 images to average. The bias is $7 \frac{\mathrm{m}}{\mathrm{s}}$. For exactly known ring centers, each can be even better than $2 \frac{\mathrm{m}}{\mathrm{s}}$. The methods could also be useful for high-resolution laser spectroscopy.
\end{abstract}

PACS $42.68 . \mathrm{Wt} \cdot 42.25 . \mathrm{Hz} \cdot 95.55 . \mathrm{Aq}$

\section{Introduction}

In atmospheric remote sensing, Doppler wind lidar (DWL) [1] is a commonly used tool for measuring the wavelength or frequency shift of light backscattered from moving atmospheric molecules and aerosols, and thus extracting information about the wind speed and direction. Coherent [2-6] or incoherent detection methods were realized. Incoherent (direct-detection) DWL has three main categories: the edge or double-edge (DE) technique [7-14], techniques based on temperature stabilized iodine vapor cells as a frequency discriminator [15-17] and the fringe-imaging (FI) technique [1822] . All three make use of instruments like Michelson [22] or (multiple) Fabry-Pérot interferometers (FPI) [14,15, 17,21], with lasers emitting at $532 \mathrm{~nm}$ or even better at $355 \mathrm{~nm}$ to obtain stronger signals from molecular backscattering. DE and FI techniques have been compared and analysed in [23,24]. A FI-type FPI was the first one to be tested on aircraft [25, 26] for wind speed measurements. FPIs are applied in scanning mode for plasma jet Rayleigh scattering measurements to determine gas temperature and velocity profiles of plasmas [27], in planar Doppler velocimetry [28] or, similar to our approach, in imaging mode to measure the flow properties by Rayleigh scattering in a small supersonic wind tunnel [29] via the locations of interference fringes [30] on a two-dimensional (2D) charge-coupled device (CCD). Among other Doppler lidar methods that use FPIs with 2D information are the image plane detector (IPD) for the Dynamics Explorer spacecraft [31], which allows for circular-pixel ringdetection, and the circle-to-line interferometer optical system (CLIO) $[32,33]$, which uses a reflective cone to convert circular fringes from a FPI into a linear series of spots on a CCD. A holographic circle-to-point converter for use with FPIs was invented, too [34]. We characterize a DWL that measures the Doppler shifts via FI in front of aircrafts using the angular displacement of Fabry-Pérot fringes [35,36].

The main focus of the article is on two novel radii evaluation strategies including a center determination, that make use of the complete 2D information given on a CCD screen in the focus behind a FPI, thus significantly reducing the noise of single one-dimensional (1D) cuts through the 2D rings. After a calibration to relate the ring radii to wavelengths and thus to Doppler shifts, we check these algorithms for their usefulness. We show that one of the methods is sufficient for DWL measurements, especially because of the low number of images necessary to average, which is fundamental for use as a velocity sensor on board of aircrafts.

Although our approach refers to that of Jenaro Rabadan et al. [26] and measurements of less than $2 \frac{\mathrm{m}}{\mathrm{s}}$ are reported there, in this context no algorithms for analyzing these $2 \mathrm{D}$ fringes 


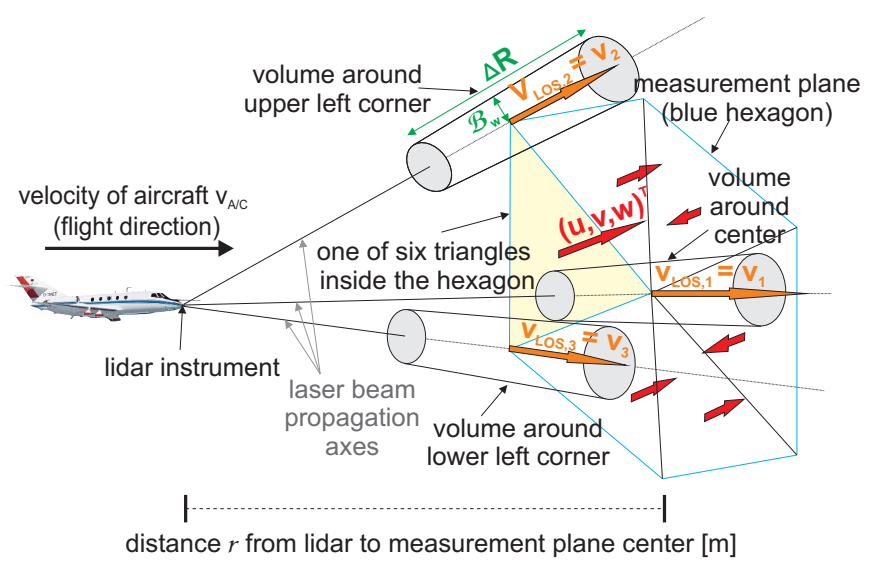

Fig. 1 Measurement principle onboard an aircraft (here DLR Falcon): see Sec. 2 for details.

in a comprehensible way have been reported yet in combination with results that show the connection of the evaluated ring radii to the Doppler shift. Furthermore we use a range bin of only $10 \mathrm{~m}$ (instead of $30 \mathrm{~m}$ in [26]) and no intensification on the CCD, but still we reach a standard deviation of less than $2 \frac{\mathrm{m}}{\mathrm{s}}$ by averaging just 20 images at $8.5 \mathrm{~km}$ altitude with the corresponding environmental conditions. A multitude of methods for $2 \mathrm{D}$ ring radii calculation of FPI fringes have been reported in literature, e.g. [37-40], some of them also including a center evaluation (e.g. $[38,40])$. While these methods are mainly suited for low noise rings in laboratory experiments, our approach is also suited for situations with very noisy rings and the necessity for subpixel accuracy.

The paper is organized as follows: after a description of the measurement geometry and a general device setup, we simulate realistic $2 \mathrm{D}$ fringe patterns including atmospheric effects and noise. Finally we calculate the ring radii (i.e. the wavelengths) of these images and assess the results.

\section{Goals and measurement geometry}

The goal is a fast and simple instrument for measurements onboard aircrafts in real-time to react to flight-safety endangering phenomena like strong gusts and wake vortices at higher flight altitude and in landing approach or at takeoffs in a distance up to $100 \mathrm{~m}$ in front of them [26]. Figures 1 and 2 illustrate the geometrical situation for the determination of a $3 \mathrm{D}$ wind vector. A laser emitter transmits one pulse of singlemode, single-frequency radiation in each so-called line-ofsight (LOS) direction under an azimuth angle $0^{\circ} \leq \theta<360^{\circ}$ and an elevation angle $0^{\circ} \leq \varphi<180^{\circ}$. For powerful laser radiation in the UV the third harmonics of Nd:YAG lasers can be used $[14,41]$. The backscattered laser light from atmospheric targets, which is Doppler-shifted, is collected by the telescope and directed to the FPI and CCD, see Fig. 3. In Fig. 1 the measurement principle is shown: Three different line-of-sight (LOS) directions, i.e. three radial LOS velocities $v_{\mathrm{LOS}, i}=v_{i}=\left|\mathbf{v}_{\mathbf{i}}\right|, i=1,2,3$, of the LOS vectors $\mathbf{v}_{\mathbf{i}}=\left(v_{x, i}, v_{y, i}, v_{z, i}\right)^{T}$ (see also Fig. 2) (orange), with their measurement volumes (range bin $\Delta R$, beam diameter $B_{\mathrm{w}}$ ) located around the center, the upper and the lower left corners of a hexagon (blue measurement plane) here, yield one 3D wind vector $(u, v, w)^{T}$ (red). The edges of the yellow triangle are the centers of the LOS-volumes, and inside each triangle homogeneity of the wind field is assumed. Thus seven LOS components may yield six 3D wind vectors, if the wind vectors are cross-correlated with each other by the center LOS-component, whose measurement may not fail in this configuration. Each scan may take only very short time and CCD data readout speed is a crucial factor. Solving an inhomogeneous linear equation system of the general form $A \mathbf{x}=\mathbf{b}$ with

$$
\left[\begin{array}{ccc}
\cos \theta_{1} \cos \varphi_{1} & \sin \theta_{1} \cos \varphi_{1} & \sin \varphi_{1} \\
\cos \theta_{2} \cos \varphi_{2} & \sin \theta_{2} \cos \varphi_{2} & \sin \varphi_{2} \\
\cos \theta_{3} \cos \varphi_{3} & \sin \theta_{3} \cos \varphi_{3} & \sin \varphi_{3}
\end{array}\right]\left[\begin{array}{c}
u \\
v \\
w
\end{array}\right]=\left[\begin{array}{c}
v_{\mathrm{LOS}, 1} \\
v_{\mathrm{LOS}, 2} \\
v_{\mathrm{LOS}, 3}
\end{array}\right],
$$

with coefficient matrix $A \in \mathbb{R}^{3 \times 3}$, unknown wind vector $\mathbf{x} \in \mathbb{R}^{3 \times 1}$ and the measured value vector $\mathbf{b} \in \mathbb{R}^{3 \times 1}$, as well as corresponding known angles $\theta_{i}$ and $\varphi_{i}(i=1,2,3)$ for each LOS component, one full 3D wind vector [42] can be determined.

Angles $\theta$ and $\varphi$ must not be too small since the vertical $\left(v_{x}\right)$ and horizontal $\left(v_{y}\right)$ components (see Fig. 2) of the LOS vectors become too small and therefore too erroneous otherwise. On the other hand, $\theta$ and $\varphi$ chosen too large destroys the possibility to calculate enough wind vectors in a plane in front of the aircraft to resolve small-scale phenomena like wake vortices. If the scatterers move towards the lidar, the backscattered radiation is shifted to a higher frequency (lower wavelength $\lambda$ ), and vice versa. Due to temporally changes of the wind, the measurement plane should be as near as possible in front of the aircraft, to fly through nearly the same wind as predicted by the FPI fringes, thus imposing a severe time limit on determining the wavelengths. The number of backscattered photons would also be too low for far-range measurements, and multiple scattering effects [43-45] could no more be neglected for CCD detectors consisting of many picture elements (pixels) where intensity is distributed and get a serious source of noise. In our simulations the measurement volume is approximately $0.05 \mathrm{~m}^{3}$ for the distances $r=56(76) \mathrm{m}$ of Table 1 and a range bin of $\Delta R=10 \mathrm{~m}$, with assumed values of $0.025 \mathrm{~m}$ for the radius of the outgoing laser beam and a divergence angle of $500 \mu \mathrm{rad}$ at full angle, resulting in a beam width of $B_{\mathrm{w}} \approx 0.08 \mathrm{~m}$ in the LOSvolume's center.

The aircraft's own movement relative to the moving particles will not be treated here, see e.g. $[26,46]$ for further details on a compensation algorithm.

\section{General device setup}

With third harmonic generation of a Nd:YAG-laser, pulses at $354.88 \mathrm{~nm}[14,41](354.7 \mathrm{~nm}$ for simulation purposes $)$ at a repetition rate of $100 \mathrm{~Hz}$ are emitted to the atmosphere. The 


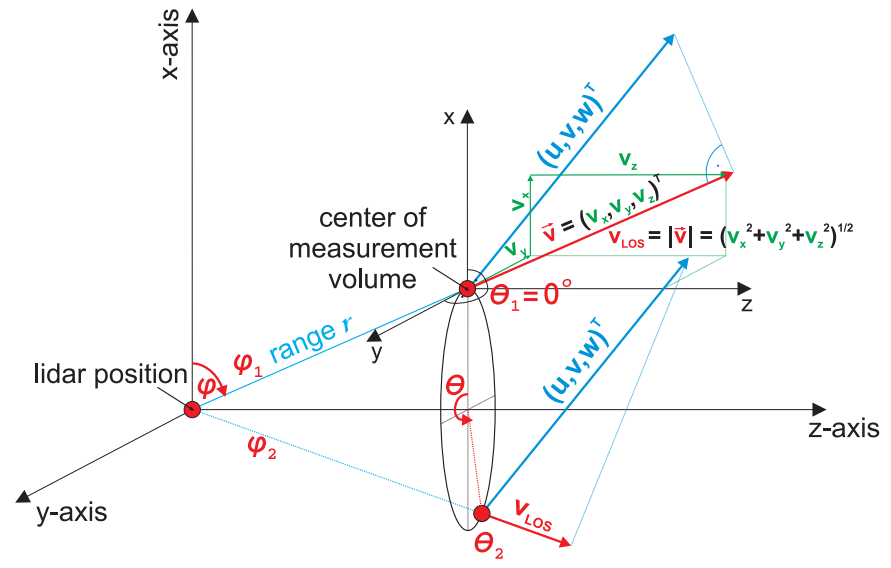

Fig. 2 Line-of-sight-velocity vector $\mathbf{v}$ (red) and its $\mathrm{x}-, \mathrm{y}-, \mathrm{z}-$ components (green) at a range $r$ for an azimuth angle $\theta$ and an elevation angle $\varphi$, and resulting absolute value $v_{\mathrm{LOS}}$ in a constant wind field $(u, v, w)^{T}$ (blue). Two realizations of $(\theta, \varphi)$ with $v_{\text {LOS }}$ are visualized here.

beams are directed to the variable LOS-directions in dependence on time. Some of the pulses are weakened and used as direct reference signals for frequency stabilization and for Doppler-shift calculations together with the backscattered light from the atmosphere. A wavemeter is required as well for stabilizing the seed laser wavelength to stay within the free spectral range (FSR) of the FPI (i.e. the same interference order) for unique wavelength assignment (accuracy of $\pm 0.002 \mathrm{~nm}$ or better required). The receiving telescope collects the backscattered photons as well as background radiation of the solar spectrum. After a field stop a fast switch or chopper between reference laser signal and backscattered signal is implemented into the optical paths, that is switched according to the time between two consecutive pulses, and whether the signal is used for referencing or for atmospheric backscattering, which delays the signal approximately two times the measurement distance. A schematical setup of the FI-receiver including only the most essential parts is shown in Fig. 3. Behind a first collecting lens a narrow-band filter blocks most of the broad solar radiation, allowing only the necessary wavelengths to propagate to an air-spaced Fabry-Pérot-étalon. Another lens collects the waves behind the FPI and focuses them to the CCD in the lens focus. This CCD is triggered by the pulse emission time and is activated and paused according to the desired measurement range. Between the backscatter signals, sometimes one pulse is used as reference signal, which then means a different gating time for the CCD. Finally the noisy backscattered images or the strong reference images are read out very quickly. Algorithms finally calculate the difference in the ring radii of a reference and a backscattered image, and thus the Doppler shift (extracting the true air speed is necessary in reality) in one LOS-direction each time.

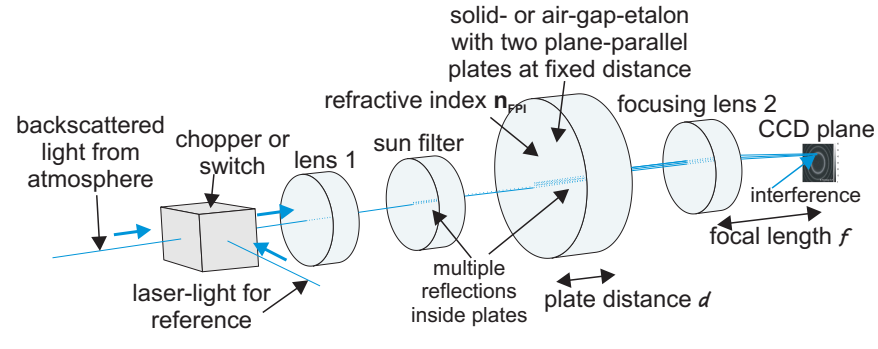

Fig. 3 Schematical setup of the FI-receiver in this study.

Table 1 Single scattering lidar equation parameters for a transmitted pulse energy of $E_{\mathrm{L}}=70 \mathrm{~mJ}$ and pulse length $\tau_{\mathrm{p}}=10 \mathrm{~ns}$ at a flight height of $8.5 \mathrm{~km}$.

\begin{tabular}{lll}
\hline$n$ & photons received at CCD & $1.3 \times 10^{7} / 2.4 \times 10^{7}$ \\
$\lambda_{\mathrm{L}}$ & center pulse wavelength & $354.7 \mathrm{~nm}$ \\
$r$ & range & $76 \mathrm{~m} / 56 \mathrm{~m}$ \\
$\beta\left(\lambda_{\mathrm{L}}, h\right)$ & backscatter coefficient & $3.104 \times 10^{-6} \mathrm{~m}^{-1} \mathrm{sr}^{-1}$ \\
$A_{\mathrm{r}}$ & area of the telescope & $0.13 \mathrm{~m}^{2}$ \\
$k$ & instrument constant & 0.15 \\
$\alpha\left(\lambda_{\mathrm{L}}, h\right)$ & extinction coefficient & $2.70 \times 10^{-5} \mathrm{~m}^{-1}$ \\
$\Delta R$ & range of atmo. volume & $10 \mathrm{~m}$ \\
\hline
\end{tabular}

\section{Fabry-Pérot ring generation including atmospheric effects and noise}

For simulation and visualization of the Fabry-Pérot-generated fringes on the CCD (see Fig. 3), a number of important properties have to be taken into account, see Table 1 . These will be discussed in detail in the following subsections and included in the FPI ring creation process for an aircraft flight height of $8.5 \mathrm{~km}$. A different approach to generate these FPI rings by ray tracing simulations [47] making use of wave properties will be described in a forthcoming publication.

\subsection{Atmospheric properties}

Multiple studies with lidars on the elastic backscattering and extinction properties for varying heights and regions have been carried out [49-52]. A reference model for the atmosphere [51,52] from airborne backscatter measurements at specific wavelengths is applied to estimate the backscatter and extinction coefficients at $355 \mathrm{~nm}$.

Rayleigh (molecular) scattering is split into a non-shifted center part, called the Cabannes line, and shifted sidebands due to (pure) rotational Raman scattering [53-58]. In this study we use the Gaussian approximation (low density of particles) of the Cabannes line for simplicity.

For our simulations the molecular backscatter coefficient $\beta_{\text {mol }}$ is derived from the Rayleigh backscatter cross section per air molecule, which was measured at a (back)scattering angle of $\pi$ at $\lambda=0.55 \mu \mathrm{m} \mathrm{[59]:} \mathrm{d} \sigma /\left.\mathrm{d} \Omega\right|_{\pi}=5.45 \times 10^{-32} \mathrm{~m}^{2} \mathrm{sr}^{-1}$. The formula [52]

$\beta_{\mathrm{mol}}(h, \lambda)=10^{-7}\left(\frac{1064 \mathrm{~nm}}{\lambda}\right)^{4.09} \exp \left(-\frac{h}{8000 \mathrm{~m}}\right) \frac{1}{\mathrm{~m} \mathrm{sr}}$, 

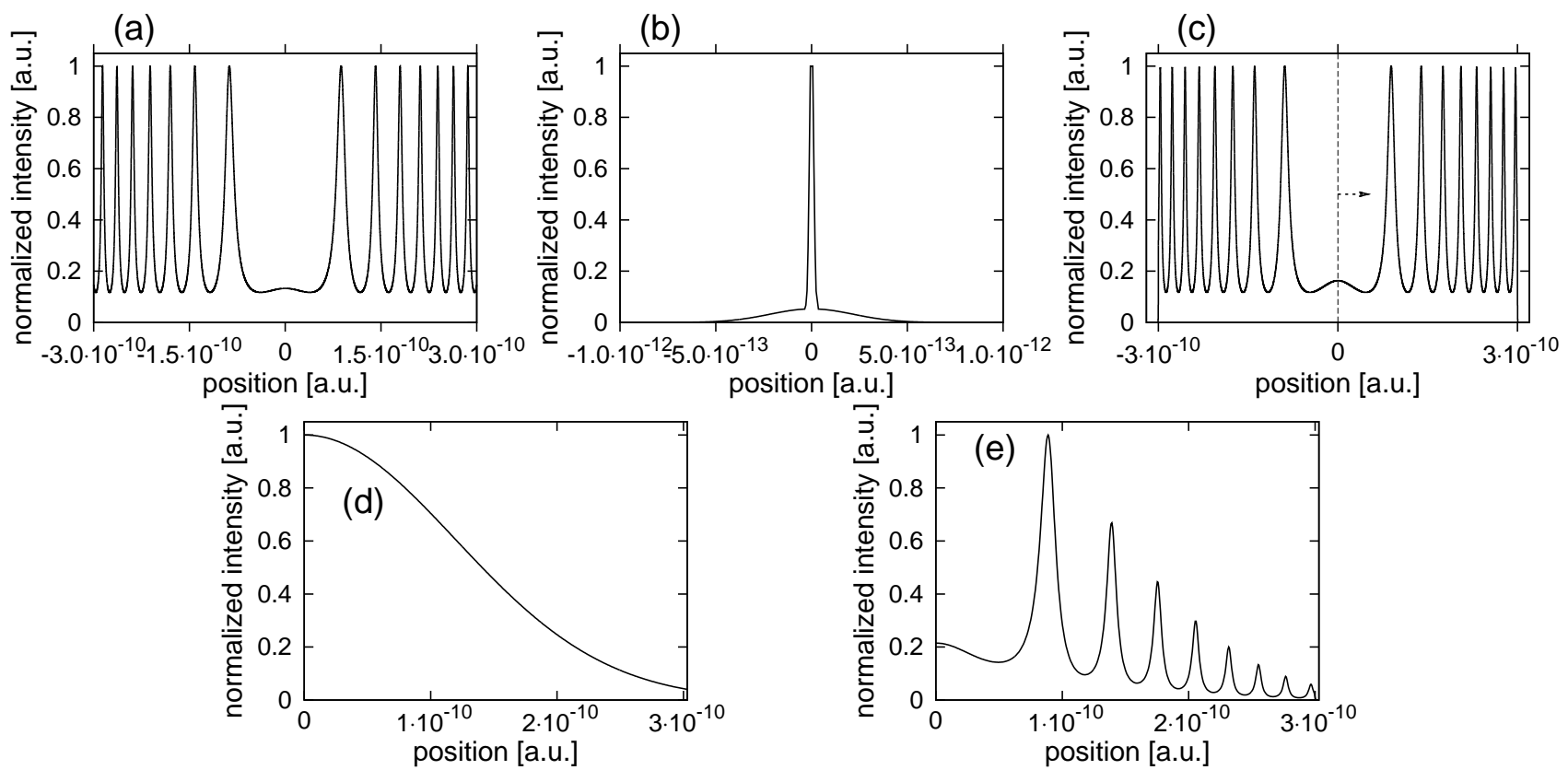

Fig. 4 Creation of FPI rings in 1D: (a) interferometric pattern calculated by the Airy function; (b) sum of Mie and Rayleigh backscattering spectra (assumed to be Gaussian-shaped); (c) convolution $\overline{C_{1}}$ (normalized to 1) of the Airy function from (a) with the composed function from (b); (d) a special normalized function $\overline{M_{\geq 0}}(\lambda)$ depending on a constant value $g$ (here $g=7 \times 10^{19}$ ); (e) product $C_{2}$ of (d) and the part right of the arrow in (c), and $C_{2}$ normalized to 1 .

yields $\beta_{\mathrm{mol}}(8500 \mathrm{~m}, 355 \mathrm{~nm})=3.08 \times 10^{-6} \mathrm{~m}^{-1} \mathrm{sr}^{-1}(\mathrm{ex}-$ ponent 4.09 instead of 4 to account for dispersion of the refractive index of air). The molecular extinction, determined by $\alpha_{\mathrm{mol}}=\beta_{\mathrm{mol}} 8 \pi / 3$, is

$\alpha_{\text {mol }}(8500 \mathrm{~m}, 355 \mathrm{~nm})=2.58 \times 10^{-5} \mathrm{~m}^{-1}[60]$.

For Mie scattering the corresponding aerosol backscatter coefficient is calculated by [61]

$$
\beta_{\text {aer }}(h, \lambda)=\beta_{0}(h, 10.6 \mu \mathrm{m})\left(\frac{10.6 \mu \mathrm{m}}{\lambda(\mu \mathrm{m})}\right)^{\alpha\left(\beta_{0}(h, 10.6 \mu \mathrm{m})\right)} \text {, }
$$

with

$$
\alpha\left(\beta_{0}(h, 10.6 \mu \mathrm{m})\right) \equiv-0.104 \ln \left(\beta_{0}(h, 10.6 \mu \mathrm{m})\right)-0.62 \text {. }
$$

So with $\beta_{0}(8-9 \mathrm{~km}, 10.6 \mu \mathrm{m})=4.3 \times 10^{-11} \mathrm{~m}^{-1} \mathrm{sr}^{-1}$ taken from the median value profile derived during a period of aerosol-depleted atmosphere between 1988 - 90 [52], we have $\beta_{\text {aer }}(8500 \mathrm{~m}, 355 \mathrm{~nm})=2.4 \times 10^{-8} \mathrm{~m}^{-1} \mathrm{sr}^{-1}$.

The aerosol extinction is a linear relationship $\alpha_{\text {aer }}=k^{\prime} \beta_{\text {aer }}$ for monodispersed spherical particles [50], with a constant lidar ratio (extinction-to-backscatter ratio) of $k^{\prime}=50$ sr assumed here, hence yielding $\alpha_{\text {aer }}(8500 \mathrm{~m}, 355 \mathrm{~nm})=1.2 \times$ $10^{-6} \mathrm{~m}^{-1}$ here.

The total extinction therefore is $\alpha=\alpha_{\text {aer }}+\alpha_{\text {mol }}$ with $\alpha(8500 \mathrm{~m}, 355 \mathrm{~nm})=2.70 \times 10^{-5} \mathrm{~m}^{-1}$ and the total backscattering $\beta=\beta_{\text {aer }}+\beta_{\text {mol with }}$

$\beta(8500 \mathrm{~m}, 355 \mathrm{~nm})=3.104 \times 10^{-6} \mathrm{~m}^{-1} \mathrm{sr}^{-1}$. The scattering ratio $R_{\beta}=\beta / \beta_{\text {mol }}$ shows the very low aerosol contribution compared to the molecular one: $R_{\beta}(8500 \mathrm{~m}, 355 \mathrm{~nm}) \approx$ 1.01 .

\subsection{Single scattering lidar equation}

The number of received photons $n$ per range gate is calculated by the single scattering lidar equation [60]

$$
n\left(\lambda_{\mathrm{L}}, r\right)=E_{\mathrm{L}} \lambda_{\mathrm{L}} \frac{\Delta R}{h c} \frac{A_{\mathrm{r}}}{r^{2}} k \beta \exp \left(-2 \int_{0}^{r} \alpha d s\right),
$$

where Planck's constant is $h=6.626 \times 10^{-34} \mathrm{~J} \mathrm{~s}$ and the speed of light is $c=2.998 \times 10^{8} \mathrm{~m} \mathrm{~s}^{-1}$. Parameters and their values for a transmitted pulse energy of $E_{\mathrm{L}}=70 \mathrm{~mJ}$ and a pulse length $\tau_{\mathrm{p}}=10 \mathrm{~ns}$ (sufficiently short for a measurement interval of $\Delta R=10 \mathrm{~m}$ ) are listed in Table 1 . The backscattering and extinction coefficients are taken for a height of $8500 \mathrm{~m}$ at a wavelength of $355 \mathrm{~nm}$ from Sec. 4.1. At a measurement distance of $r=76 \mathrm{~m}$ approximately 13 million photons reach the CCD, with an instrument constant of $k=$ 0.15 and a range bin of $\Delta R=10 \mathrm{~m}$, while it is 24 million photons at a shorter distance of $r=56 \mathrm{~m}$. The telescope radius is $0.2 \mathrm{~m}$, i.e. $A_{r}=0.13 \mathrm{~m}^{2}$. Typical values from novel DWL instruments were taken for these considerations [14].

The instrument constant $k=0.15$ is the result of a product of four assumed optimal values, that may be realizable today. These are the photon emission factor and the factors of receive, sunfilter and Fabry-Pérot transmission. The laser's photon emission factor to the atmosphere is 0.98 . The backscattered signal is collected by the telescope, reflected by mirrors and passes the front optics (fiber coupling or free optical path propagation) with a receive transmission of 0.65 . Field stops in the front optics limit the telescope's FOV to $1 \mathrm{mrad}$ at full angle in order to minimize the solar background. Pulse energy has to be increased or the measurement distance has to 
be reduced if this factor is lower than 0.65 in reality. At a FWHM of $0.5 \mathrm{~nm}$ the sunfilter transmits a factor of 0.95 . For a low reflectivity of 0.70 of the FPI plates the losses inside the étalon are relatively low, not considering absorption of the plates. Depending on the width of the fringes, the corrected transmission factor of the étalon onto the CCD, calculated by integrating the intensity columns of patterns like the one shown in Fig. 4(e) and dividing them by intensity columns of maximum value 1 , may slightly vary around 0.25 . So 0.25 was chosen as a mean value.

\subsection{Mie and Rayleigh scattering}

Simulations were performed with Gaussian models of Mie and Rayleigh scattering. Mie and Rayleigh scattering functions in our case are needed for a convolution to create rings of different widths depending on temperature and pressure or heights according to US Standard atmosphere [62]. The calculations will be valid for all kinds of FPI rings with symmetrically shaped peaks over a background.

The first step is carried out one-dimensional. The Rayleigh scattering line shape is governed by broadening which results in the following Gaussian line profile function $[50,60]$ centered around $\lambda_{\mathrm{L}}$ :

$$
I_{\mathrm{R}}(\lambda)=\frac{1}{\sqrt{2 \pi \sigma_{\mathrm{R}}^{2}}} \exp \left(-0.5 \frac{\left(\lambda-\lambda_{\mathrm{L}}\right)^{2}}{\sigma_{\mathrm{R}}^{2}}\right),
$$

where $\sigma_{\mathrm{R}}$ denotes the standard deviation of the Rayleigh spectrum given by

$$
\sigma_{\mathrm{R}}\left(m_{\mathrm{air}}\right)=\frac{2 \lambda_{\mathrm{L}}}{c} \sqrt{\frac{k_{\mathrm{B}} T N_{\mathrm{A}}}{m_{\text {air }}}},
$$

where $m_{\text {air }}=2.9 \times 10^{-2} \mathrm{~kg} / \mathrm{mol}$ is the mean molecular air mass, $\lambda_{\mathrm{L}}$ is the laser wavelength, $k_{\mathrm{B}}=1.38 \times 10^{-23} \mathrm{~J} / \mathrm{K}$ the Boltzmann constant, $T(8500 \mathrm{~m})=232.9 \mathrm{~K}$ the temperature, $c$ the speed of light, and $N_{\mathrm{A}}=6.023 \times 10^{23} \mathrm{~mol}^{-1}$ the Avogadro constant.

The laser beam intensity is assumed to have a Gaussian shaped spectral distribution [63]:

$$
I_{\mathrm{L}}(\lambda)=\pi \tau_{\mathrm{p}}^{2} \exp \left(-0.5(\Delta \omega)^{2} \tau_{\mathrm{p}}^{2}\right),
$$

with pulse length $\tau_{\mathrm{p}}=10 \mathrm{~ns}$ and frequency interval $\Delta \omega=$ $2 \pi c\left(\lambda-\lambda_{\mathrm{L}}\right) / \lambda_{\mathrm{L}}^{2}$. The step resolution $\lambda$ is used for modeling. The full width at half maximum of (8) is $\Delta \lambda_{\mathrm{LFWHM}_{\mathrm{FW}}}=$ $2\left|\lambda_{\text {I,max }}-\lambda_{\mathrm{LFWHM}_{\mathrm{FW}}}\right|$, with the $\lambda$-value of peak intensity (the center) $\lambda_{\mathrm{I}, \max }$ and the distance $\lambda_{\mathrm{L}_{\mathrm{FWHM}}}$ to it. The aerosol (Mie) scattering spectrum $I_{\mathrm{M}}(\lambda)$, governed by the laser profile, looks like that of (6), replacing only $I_{\mathrm{R}}$ by $I_{\mathrm{M}}$ and $\sigma_{\mathrm{R}}$ by $\sigma_{\mathrm{M}}$, with

$$
\sigma_{\mathrm{M}}=\frac{\Delta \lambda_{\mathrm{L}_{\mathrm{FWHM}}}}{\sqrt{8 \cdot \ln 2}},
$$

where $\Delta \lambda_{\mathrm{L}_{\mathrm{FWHM}}}$ is the full width at half maximum (FWHM) of the Gaussian laser spectrum mentioned afore. The Mie peak is added to the broadband Rayleigh part, see Fig. 4 (b). The shape of this pattern is important, hence the diagrams with arbitrary units illustrate the principle.

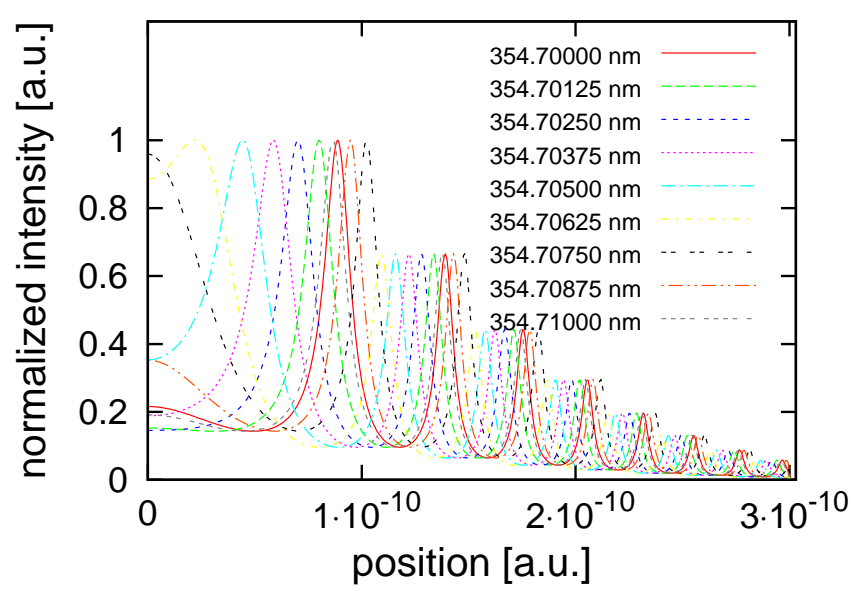

Fig. 5 Wavelength dependence of the 1D fringes for one full FSR between $354.70 \mathrm{~nm}$ and $354.71 \mathrm{~nm}$.

\subsection{FPI transmission}

The transmitted intensity of a FPI is described by the classical Airy function (AF) without absorption at the plates $[35,36$, 48]

$$
I_{\mathrm{A}, \mathrm{T}}(\delta)=I_{0} \frac{1}{1+F \sin ^{2}(0.5 \delta)},
$$

with $I_{0}$ the maximum intensity behind the FPI (on a CCD), $F=\pi \sqrt{R} /(1-R)$ the reflectivity finesse $(F=8.76$ for a plate reflectivity of $R=0.70$ here) and $\delta$ the geometrical phase difference according to the formula

$$
\delta=\frac{4 \pi n_{\mathrm{F}} d \cos \theta_{\mathrm{F}}}{\lambda},
$$

where $n_{\mathrm{F}}=1$ is the refractive index in between two airspaced plates at distance $d=6.5 \mathrm{~mm}$ with varying incident angles $\theta_{\mathrm{F}}$ of $-20.71 \mathrm{mrad} \leq \theta_{\mathrm{F}}(\lambda) \leq 20.71 \mathrm{mrad}$, corresponding to seven fringe orders (see Fig. 4(a)). These were determined by values $354.40 \mathrm{~nm} \leq \lambda \leq 355.00 \mathrm{~nm}$ in the equation $\theta_{\mathrm{F}}(\lambda)=\left(\lambda-\lambda_{\mathrm{L}}\right) /\left(\lambda_{\mathrm{L}} 2 \pi n_{\mathrm{F}} d\right)$. To have a high throughput from the FPI to the CCD, $R$ should be low, which is specific to such instruments $[23,24]$. The optimal values in McGill and Spinhirne [24] with $d=9.5 \mathrm{~mm}$ and $R=0.715$ were very similar in their order of magnitude to the chosen ones, although their FI-method is different from the one shown here. Our simulated fringe patterns had an optimal shape at $d=6.5 \mathrm{~mm}$ and $R=0.70$, to get two full $2 \mathrm{D}$ rings on a CCD later on. It will be advantageous for the signal processing algorithms to have broad and not too steep FPI ring shapes, see Secs. 5 and 6. The minimum FSR is $0.0097 \mathrm{~nm}$ according to $\mathrm{FSR}=\lambda^{2} /\left(2 n_{\mathrm{F}} d\right)$. The result is shown in Fig. 4 (a).

\subsection{Convolutions in $1 D$, extension from $1 D$ to $2 D$}

First a convolution $C_{1}$ of the $\operatorname{AF} I_{\mathrm{A}, \mathrm{T}}(\delta)=I_{\mathrm{A}, \mathrm{T}}(\lambda)$ with the composed function of $I_{R}$ and $I_{M}$, called $I_{\mathrm{M}}+I_{\mathrm{R}}$, creates an 
underground below the AF (Fig. 4 (c)) and may broaden the final fringes via

$$
\begin{aligned}
C_{1}(\lambda) & =\left(I_{\mathrm{A}, \mathrm{T}} *\left(I_{\mathrm{M}}+I_{\mathrm{R}}\right)\right)(\lambda) \\
& =\int_{\mathbb{R}} I_{\mathrm{A}, \mathrm{T}}(x)\left(I_{\mathrm{M}}+I_{\mathrm{R}}\right)(\lambda-x) d x,
\end{aligned}
$$

where $*$ denotes the convolution operator. The segment width has to be equal for $I_{\mathrm{A}, \mathrm{T}}(\lambda)$ and $I_{\mathrm{M}}+I_{\mathrm{R}}$. Then the maximum of $C_{1}$ is determined and set to the value 1 ; the whole graph is stretched or jolted, see Fig. 4 (c), resulting in the function $\overline{C_{1}}$.

A decay of the intensity to the points more distant from the ring center is observed in reality, see [26,33,39,40,48]. This is here accomplished by multiplication of $\overline{C_{1}}$, taking only the points right of the ring center (arrow in Fig. 4 (c)), with the branch to the right of the maximum of a special function

$$
M(\lambda)=\sqrt{\frac{g}{2 \pi}} \exp \left(-\frac{g \lambda^{2}}{2}\right)
$$

with a constant $g=7 \times 10^{19}$ (appropriate for the dimensions of the position-axis), that was picked here due to its decay property. $M(\lambda)$ has no physical meaning and is used to model different steepnesses of peak decays by varying $g$ (Fig. 4 (d)). Only $\lambda$-values greater zero are taken and the maximum of $M$ is normalized to 1 , resulting in a function $\overline{M_{\geq 0}}(\lambda)$. The product is $C_{2}(\lambda)=\overline{C_{1}}(\lambda) \cdot \overline{M_{\geq 0}}(\lambda)$, which is normalized to a rotational-symmetric function $\overline{C_{2}}(\lambda)$ around 0 (Fig. 4 (e)).

Fig. 5 shows the wavelength-dependence of fringe patterns like the one shown in Fig. 4 (e) over one full FSR. For the wavelength-determination algorithms it will be advantageous to have the first peak away from the center as far as possible, to have more pixels to average later on. A wavelength of $\lambda=354.70000 \mathrm{~nm}$ (i.e. the center wavelength in our simulations) is therefore best suited in this case, while $\lambda=354.70625 \mathrm{~nm}$ with the first peak at the center would be a bad choice. The useful part of the FSR for the measurements should therefore be limited to $\pm 0.002 \mathrm{~nm}$ around the center wavelength.

From that (Figs. 4(e) and 5) a 2D, rotational-symmetric pattern of non-equidistant rings can be created. Depending on the width of the convolving function $I_{\mathrm{M}}+I_{\mathrm{R}}$ a quadratic image of equal number of points with equidistant segment widths on the $x$ - and $y$-axes arises, each tuple $(x, y) \in \mathbb{R}^{2}$ being a pixel. The number of points on each axis after convolutions in 1D usually was between 506 and 541. In 2D the number of points on each axis is doubled by symmetry around 0 . CCD imagers normally are non-quadratic, so on each axis a number of points are dropped (see Figs. 6 (a), (b)), and instead of $1010 \times 1010$ pixels any lower dimension can be chosen; in our case it is $961 \times 781$ pixels (see Figs. 6 (c), (d)), since odd numbers yield a unique center pixel at $(0,0)$ and for the algorithms a pixel number as high as possible should be aimed at.

\subsection{Noise modeling by random number generation}

A PCO dicam pro intensified-CCD (ICCD) camera [64] and its specifications may serve as a sample for a suitable imaging device. Most of the parameters for the simulations are chosen in the order of magnitude of this camera's specifications. A model of this series was used for DWL measurements in an aircraft [25]. However, a CCD's noise properties were modeled here, which means an excess noise factor $(\mathrm{ENF})$ of $\mathrm{ENF}_{\mathrm{CCD}} \approx 1$ (i.e. gain factor of 1 , no excess noise) [65], while it is $\mathrm{ENF}_{\mathrm{ICCD}} \approx 2$ for an ICCD with gain factors of 100 and higher [65]. Intensification is excluded for simplicity and to simulate a worst-case scenario in this study. Despite the fact that the ICCD shows a larger ENF, this device usually provides a greater signal-to-noise ratio (SNR) than a CCD, as shown by Carranza et al. [66] for a spectral range around $400 \mathrm{~nm}$. The shortest shutter of the ICCD camera is as low as $3 \mathrm{~ns}$, thus realization of intervals of $67 \mathrm{~ns}$ (range gate $\Delta R=10 \mathrm{~m}$ ) is possible. One hundred pulses per second means 100 CCD images (see Sec. 3) and in this way about $10 \mathrm{~ms}$ time to readout the data of one image, before this step is repeated. The laser is linked to the CCD to trigger the CCD shutter with the moment of pulse emission.

Three main sources of noise distort the optimal shape of the rings shown in Figs. 6 (a) and (b). For the reference signal speckle noise is relevant, at insignificantly low photon noise. The backscattered signal comprises neglectable speckle noise, while photon noise is dominating. The CCD's readout noise occurs for both signal types.

Dark current noise can be ignored owing to the short gate time of $67 \mathrm{~ns}$ and the narrow-band filter in the receiver. Usual dark currents are lower than 100 electrons per second for each pixel, so it should be maximum $0.67 \times 10^{-6}$ electrons per pixel in $67 \mathrm{~ns}$ in our case. Solar noise can also be excluded from modeling, see Sec. 4.7.

Artificial creation of noise implies using a modern, nonstandard, fast random number generator (RNG) like Ran of Numerical Recipes [67] for uniform deviates with sufficiently long period to create variable values without repetition. The seed values to start the RNG must be chosen differently for every picture.

CCDs are arrays of light-sensitive photodiodes that generate and store electrons from photons with a certain quantum efficiency $\eta$ ( $\eta \approx 0.21$ for dicam CCD at $355 \mathrm{~nm}$ ) [68]. We define the number of signal (photo) electrons $\left(\mathrm{e}^{-}\right)$generated by a number $n$ of backscattered (calculated by (5)) or direct photons by

$$
n_{\mathrm{sg}}=\eta n
$$

These $n_{\text {sg }}$ signal electrons are distributed among all the pixels of the CCD according to the pattern of Fig. 6 (a) and their number on each pixel is rounded.

Photon (shot) noise arises from statistical fluctuations in the number of photons at low count rates on the detector and is described by a Poisson statistic $[69,70]$. If the number of 

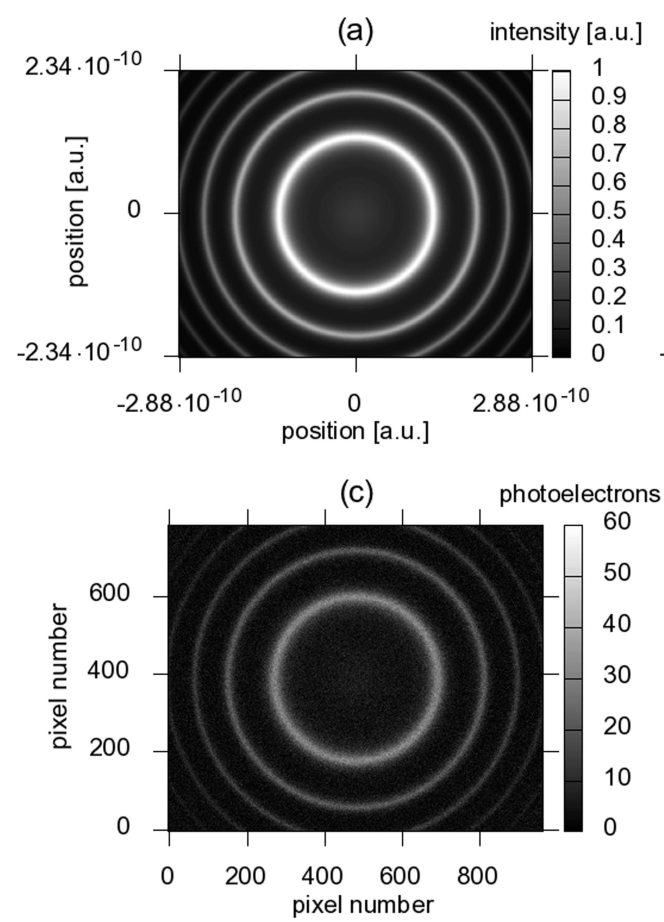

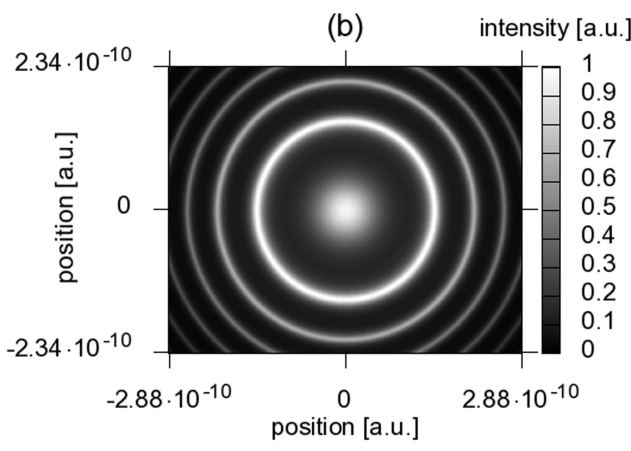

(d)

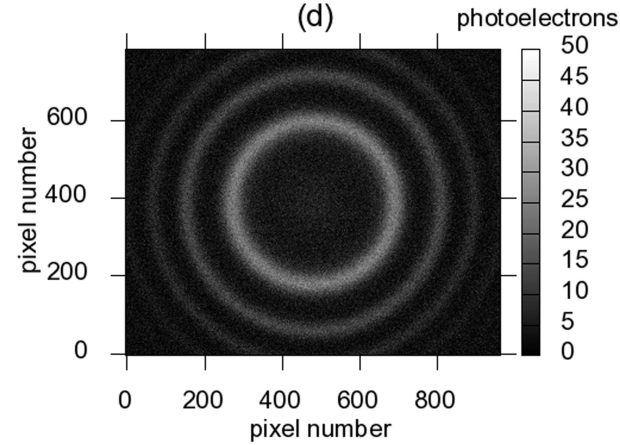

Fig. 6 Creation of FPI rings in $2 \mathrm{D}$ on a CCD (continued from Fig. 4): (a) 2D, rotational-symmetric pattern of non-equidistant rings at $\lambda=354.7000 \mathrm{~nm}$ after nonquadratic area reduction from a square to a rectangular shaped CCD image; (b) the same as (a) for $\lambda=354.7075 \mathrm{~nm}$; (c) pattern at $\lambda=354.7000 \mathrm{~nm}$ after restructuring of the positions in (a) to pixel numbers, and distribution of photoelectrons onto the pixels of the $\mathrm{CCD}$ image; (d) the same as (c) for a broad ring structure due to convolution. signal electrons on a single pixel with index $i$ is $n_{\mathrm{sg}, i}$, then

$$
\overline{n_{\mathrm{sg}}}=\frac{1}{m \cdot n} \sum_{i=1}^{m \cdot n} n_{\mathrm{sg}, i}
$$

denotes the average signal electrons on each pixel (the mean and variance of a Poisson deviate), where $m$ and $n$ are the $x$ and $y$-axes dimensions (e.g. $m=961$ and $n=781$ pixels in Fig. 6), respectively. In order to simulate the number of $\mathrm{e}^{-}$, the Ran-RNG [67] was used to genereate Poisson-distributed random numbers by the so-called rejection-method [67]. The probability distribution is

$$
\mathbb{P}_{\text {Poi }, \overline{n_{\mathrm{sg}}}}\left(X_{\mathrm{sg}, i}=n_{\mathrm{sg}, i}\right)=\frac{\overline{n_{\mathrm{sg}}} n_{\mathrm{sg}, i}}{\left(n_{\mathrm{sg}, i}\right) !} \exp \left(-\overline{n_{\mathrm{sg}}}\right),
$$

which denotes the probability, that the random numbers (variables) $X_{\mathrm{sg}, i}$ will take the values (realizations) $n_{\mathrm{sg}, i}$ [70].

Gamma-distributed random numbers serve for characterization of speckle noise, with parameters $s_{i}$ for the number of speckle grains averaged on the $i$-th pixel which can be measured for each pixel; we took an average value of $\bar{s}=10.0$ for all the $s_{i}$, since similar values were measured [70]. The probability distribution is

$\mathbb{P}_{\Gamma}\left(X_{\mathrm{sg}, i}=n_{\mathrm{sg}, i}\right)=\frac{1}{\Gamma\left(s_{i}\right)}\left(\frac{s_{i}}{\overline{n_{\mathrm{sg}}}}\right)^{s_{i}} n_{\mathrm{sg}, i}^{s_{i}-1} \exp \left(-s_{i} \frac{n_{\mathrm{sg}, i}}{\overline{n_{\mathrm{sg}}}}\right)$,

with a mean of $\overline{n_{\mathrm{sg}}}$, a variance of $\left({\overline{n_{\mathrm{sg}}}}^{2}\right) / s_{i}$, and a Gammafunction $\Gamma(\cdot)$ that is commonly defined.

The CCD's readout noise obeys a Gaussian-distribution, thus normal deviates created by the ratio-of-uniforms method
[67] are taken, with a probability distribution

$$
\mathbb{P}_{\mathcal{N}\left(\mu, \sigma^{2}\right)}\left(X_{\mathrm{sg}, i}=n_{\mathrm{sg}, i}\right)=\frac{1}{\sqrt{2 \pi \sigma^{2}}} \exp \left(-\frac{\left(\mu-n_{\mathrm{sg}, i}\right)^{2}}{2 \sigma^{2}}\right),
$$

with the mean $\mu=0$ and the variance $\sigma^{2}$ ( $\sigma$ may vary depending on the type of CCD; here $\sigma=5.0$ was chosen, similar to a measured value for a CCD [70]).

For simulations of backscatter signals (as shown in Fig. 6), the final number of charge carriers on a pixel $i$ is

$$
n_{\mathrm{C}, \mathrm{i}}=\mathrm{RN}_{\mathrm{Poi}, \mathrm{i}}+\mathrm{RN}_{\mathcal{N}, \mathrm{i}}
$$

where $R N_{P o i, i}$ and $R N_{\mathcal{N}, i}$ are the random numbers including photon and readout noise, respectively, that were created using the probability distributions specified in (16) and (18). For reference signals or for calibration we have

$$
n_{\mathrm{C}, \mathrm{i}}=\mathrm{RN}_{\Gamma, \mathrm{i}}+\mathrm{RN}_{\mathcal{N}, \mathrm{i}},
$$

where $\mathrm{RN}_{\Gamma, \mathrm{i}}$ are the random numbers including speckle noise related to (17). Thus uncorrelated noise sources (especially photon and speckle noise [71]) can be assumed, i.e. the random numbers are independent and can be summed. Negative numbers of $n_{\mathrm{C}, \mathrm{i}}$, which may occur due to the normal deviates, are set to zero (numbers of $\mathrm{e}^{-}$lower 0 are impossible). Results of differently broad rings for backscatter signals at $r=56 \mathrm{~m}$ (24 million photons) due to modeling, including noise, are shown in Figs. 6 (c) and (d).

\subsection{Solar background}

If the sky is clear, i.e. at lack of aerosols, the lidar will detect solar radiance (direct and scattered by molecules) besides the 


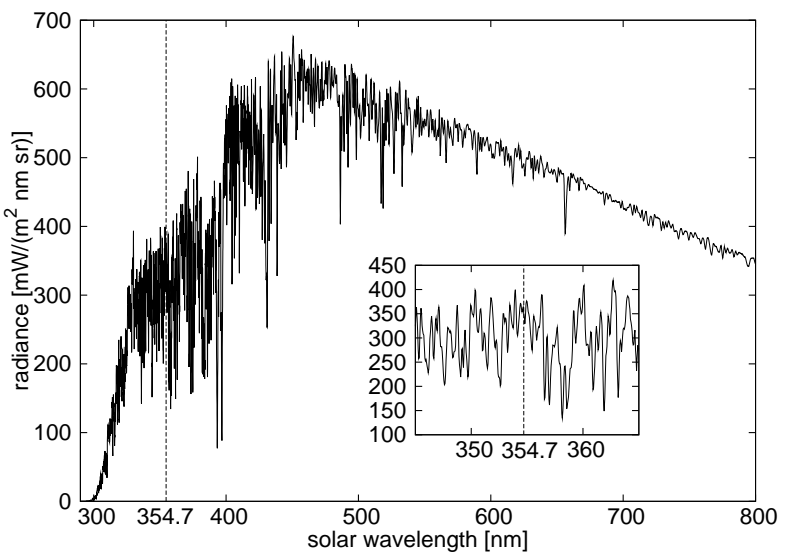

Fig. 7 Received solar spectrum from $290-800 \mathrm{~nm}$.

backscattered laser photons. The contribution of this source of noise, called solar background, is modeled using the radiative transfer software package libRadtran [72]. At an altitude of $10000 \mathrm{~m}$, where the distance sun-earth is smaller and no clouds extinct the radiance, the number of solar photons should be reduced by a narrow-band transmissive filter (FPI étalon), that eliminates photons with wavelengths lower or higher than the laser frequency or the wavelength-shifted received light.

In the situation of Figs. 1 and 2 we assume that a lidar emits at an disadvantageous elevation angle of $\varphi=110^{\circ}$ in an airplane flying at a constant level; so the laser points $20^{\circ}$ downwards in reference to the airplane propagation axis. A worst-case is considered with a maximum surface albedo of 1.0, a ozone column scaled to 300 Dobson units, a midsummer day on the northern hemisphere with solar zenith angle of $0^{\circ}$, solar azimuth angle of $0^{\circ}$ (i.e. sun in the south), a sensor viewing zenith angle of $20^{\circ}$ downwards and a sensor azimuth angle of $0^{\circ}$ (sensor in the north, so it is directed to the south where the sun is) at an altitude of $10 \mathrm{~km}$ for US Standard atmosphere. The sun intensity is even slightly higher for this case than for a lidar pointing $20^{\circ}$ upwards, since surface reflectance adds to direct sun radiance due to no clouds. The atmosphere radiance (intensity) $L_{\text {atmo }}$ in $\mathrm{W} /\left(\mathrm{m}^{2} \mathrm{~nm}\right.$ sr) for the specific wavelengths calculated by libRadtran are linearly interpolated to intervals of $0.01 \mathrm{~nm}$ stepwidth. Fig. 7 shows the received spectrum in $\mathrm{mW} /\left(\mathrm{m}^{2} \mathrm{~nm} \mathrm{sr}\right)$ at the telescope at the specified conditions for a range of $290-800 \mathrm{~nm}$.

The sunfilter-reduced background on the CCD obeys the formula

$$
P_{\text {backgr }}(\lambda)=L_{\text {atmo }}(\lambda) A_{\mathrm{r}} \pi(0.5 \Theta)^{2} B_{\text {eff }}(\lambda) \tau_{\mathrm{f}} \tau_{\mathrm{t}},
$$

with the telescope area $A_{\mathrm{r}}=0.13 \mathrm{~m}^{2}$, instrument field of view (full angle) of $1 \mathrm{mrad}$, telescope transmission factor (instrument constant without sun filter) $\tau_{\mathrm{t}}=0.16$ and the product $B_{\text {eff }}(\lambda) \tau_{\mathrm{f}}$ (maximum value of $\alpha_{f}=0.95$ at $354.7 \mathrm{~nm}$, see below) of a filter and its transmission factor. For the short time interval of measurement the energy is $\Delta E(\lambda)=P_{\text {backgr }}(\lambda) \Delta t=n E_{\mathrm{ph}}$ with a number of photons $n(\lambda)$ and the single photon's energy $E_{\mathrm{ph}}(\lambda)=h c \lambda^{-1}$ with Planck's constant $h$ and distributed values of $\lambda$.

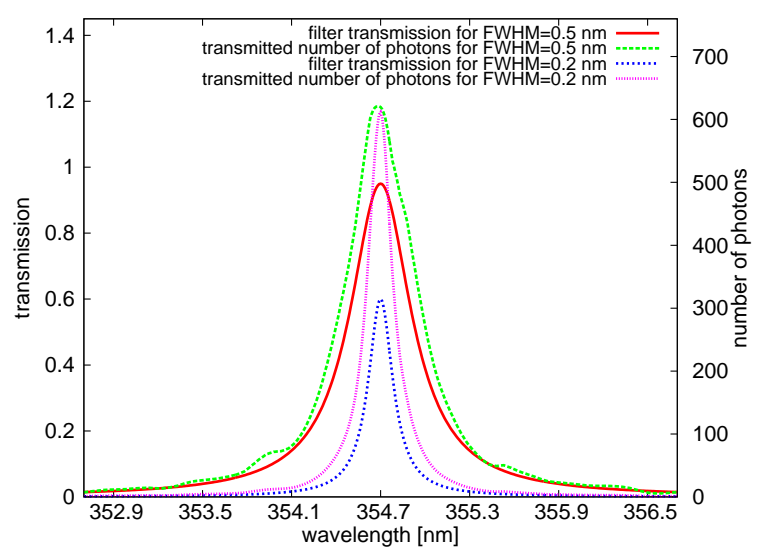

Fig. 8 Two examples for solar filters with $\Delta \lambda=0.5 \mathrm{~nm}\left(\alpha_{f}=\right.$ $0.95)$ and $\Delta \lambda=0.2 \mathrm{~nm}\left(\alpha_{f}=0.60\right)$, respectively, and the resulting $\lambda$-distributed number of photons hitting the CCD at a resolution of $0.01 \mathrm{~nm}$.

For a FPI étalon a Lorentzian function can be used as an approximation to model the filter function for a single étalon fringe $[71,73]$, with the filter transmission

$$
I_{\mathrm{T}, \mathrm{f}}(\lambda)=\alpha_{f}\left(1+\frac{\lambda-\lambda_{\mathrm{c}}}{0.5 \Delta \lambda}\right)^{-1} \in\left(0, \ldots, \alpha_{f}\right],
$$

where $\alpha_{f}$ is the assumed peak transmission factor of the filter, $\lambda_{\mathrm{c}}=354.7 \mathrm{~nm}$ the center wavelength and $\Delta \lambda$ the full width at half maximum (FWHM) of the filter. The resulting number of $\lambda$-distributed photons for two cases is shown in Fig. 8. Due to a low exposure time $\Delta t=6.7 \times 10^{-8} \mathrm{~s}$ of the instrument for a range bin of $10 \mathrm{~m}$, the number of solar photons onto the CCD is neglectably small $(n<45000$ for $\Delta \lambda=0.5 \mathrm{~nm}$ with $\alpha_{f}=0.95$ and $n<19000$ for $\Delta \lambda=0.2 \mathrm{~nm}$ with $\left.\alpha_{f}=0.60\right)$. Solar photons are equally distributed on the CCD, thus only affecting the background line but not the shape of the fringe peaks [24]. Concerning the instrument factor $k=0.15$ the peak transmissions of 0.95 (or 0.6) have only to be taken into account for the backscattered laser photons since the interesting $\lambda$-bandwidth around $\lambda_{\mathrm{c}}=354.7 \mathrm{~nm}$ is less than the FSR of $0.01 \mathrm{~nm}$. Some solar photons may also not intersect the CCD-area and will be lost in the front optics, the FPI-étalon or behind the FPI because of absorption or exceeding the allowed angle range. Measurements [26] indicated only small sun radiance influence at a height of about $12000 \mathrm{~m}$ compared to the total received intensity. With a number of definitely less than 0.1 photons on a single pixel and equal distribution, solar noise can be excluded from further analysis.

\section{Calculation of ring radii and wavelengths}

This section is devoted to the evaluation of ring radii and the absolute wavelengths, and their differences. The center of noisy 2D FPI fringe images is determined, which is unknown and may change from one image to the other due to onboard vibrations and temperature fluctuations. For the exact determination of the length of a line, its starting point has 


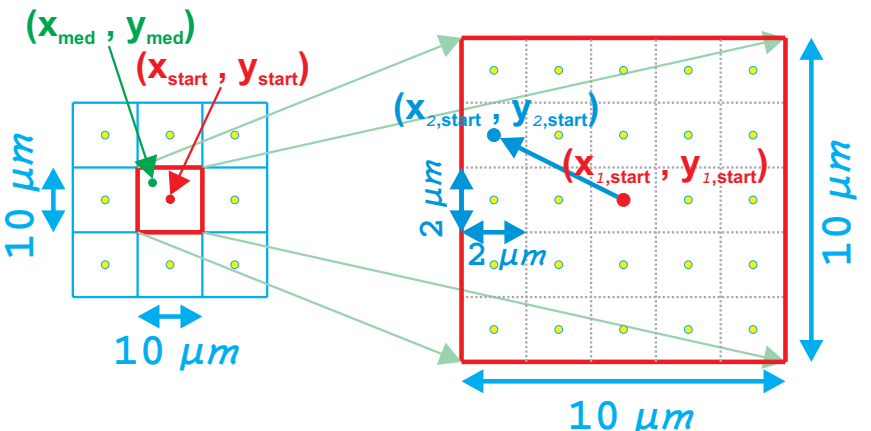

Fig. 9 Starting from a medium estimate $\left(x_{\text {med }}, y_{\text {med }}\right)$ the minimum distant pixel center $\left(x_{\text {start }}, y_{\text {start }}\right)$ is determined. Then a square neighborhood of pixels with $10 \mu \mathrm{m}$ sidelength around $\left(x_{\text {start }}, y_{\text {start }}\right)$ is defined, and $\left(x_{1, \text { start }}, y_{1, \text { start }}\right)$ is the calculated best suited one as a center. This procedure can be repeated in the subpixel area, so that a subdivision of the pixel around $\left(x_{1, \text { start }}, y_{1, \text { start }}\right)$ yields the absolutely best suited center $\left(x_{2, \text { start }}, y_{2, \text { start }}\right)$.

to be known exactly. This principle also applies for ring radii, and a center evaluation is necessary, since for a LOS-velocity bias of $1 \frac{\mathrm{m}}{\mathrm{s}}$, the radii have to be determined with a resolution better than $\frac{1}{40}$ of a pixel side length of $10 \mu \mathrm{m}$, which is also indispensable for a good calibration of the instrument. Two different methods are then applied to the images created in Sec. 4 (see Fig. 6) to reduce the noise and transform them to $1 \mathrm{D}$. Finally we are able to calculate the exact radius position by a simple fitting procedure. This approach is used for a simulated calibration, yielding relations between radii and wavelengths, and this way the bias and standard deviation of evaluation can be tested.

\subsection{Center evaluation of fringe pattern}

A well known center, especially when the ring is not exactly in the middle of the CCD image zone, is the basis for a good precision, i.e. low bias and standard deviation. Let $x_{i}$ be the $m$ and $y_{j}$ be the $n$ equidistant points on the $x$-axis and the $y$-axis, respectively, which yield pixels $\left(x_{i}, y_{j}\right) \in \mathbb{R}^{2}$ with a pixel width of $\Delta x=\Delta y=10 \mu \mathrm{m}(m=961$ and $n=781$ here), which was resized from the width of $6 \times 10^{-13}$ [a.u.] of an interval during the construction process in Sec. 4. First the center of mass of all pixels is estimated (so that the following searches for the best center point can be curtailed to a relatively small area later on), using the sum $S$ of the number of charge carriers $n_{\mathrm{C}}\left(x_{i}, y_{j}\right)$ of a CCD image with

$$
S=\sum_{i=1}^{m} \sum_{j=1}^{n} n_{\mathrm{C}}\left(x_{i}, y_{j}\right)
$$

and weighting their positions on the $x$ - and $y$-axis:

$x_{\mathrm{w}}=\sum_{i=1}^{m} x_{i} \sum_{j=1}^{n} n_{\mathrm{C}}\left(x_{i}, y_{j}\right)$ and $y_{\mathrm{w}}=\sum_{j=1}^{n} y_{j} \sum_{i=1}^{m} n_{\mathrm{C}}\left(x_{i}, y_{j}\right)$, resulting in a medium estimate $\left(x_{\text {med }}, y_{\text {med }}\right)$ with $x_{\text {med }}=$ $x_{\mathrm{w}} / S$ and $y_{\text {med }}=y_{\mathrm{w}} / S$. Then search for the minimum distance of $\left(x_{\text {med }}, y_{\text {med }}\right)$ to a pixel center $\left(x_{i}, y_{j}\right)$, i.e. find the best suited $i$ and $j$ for

$\left(x_{\text {start }}, y_{\text {start }}\right)=\left(\min _{i=1, \ldots, m}\left|x_{i}-x_{\text {med }}\right|, \min _{j=1, \ldots, n}\left|y_{j}-y_{\text {med }}\right|\right)$.

Thereafter a number of pixels in a square structure are defined around the estimated center $\left(x_{\text {start }}, y_{\text {start }}\right)$. In Fig. 9 it is a $m_{1} \times n_{1}=3 \times 3$ matrix with $m_{1}, n_{1} \in \mathbb{N}$. For these pixels with $10 \mu \mathrm{m}$ side length calculate the minimum distance from the center points of each of the 9 pixels (tiny yellow points in Fig. 9) to the edges of the whole CCD image by

$$
\begin{aligned}
& x_{\text {mindist }}=\min _{i_{1}=1, \ldots, m_{1}}\left(\left|x_{i_{1}, \text { start }}-x_{\min }\right|,\left|x_{i_{1}, \text { start }}-x_{\max }\right|\right) \\
& y_{\text {mindist }}=\min _{j_{1}=1, \ldots, n_{1}}\left(\left|y_{j_{1}, \text { start }}-y_{\min }\right|,\left|y_{j_{1}, \text { start }}-y_{\max }\right|\right),
\end{aligned}
$$

where $\left(x_{i_{1}, \text { start }}, y_{j_{1}, \text { start }}\right)$ are the pixel points in the neighborhood of $\left(x_{\text {start }}, y_{\text {start }}\right)$ (with $\left(x_{\text {start }}, y_{\text {start }}\right)$ included $), x_{\min }$, $y_{\min }$ the minimum edges, $x_{\max }, y_{\max }$ the maximum edges, and $m_{1}, n_{1} \in \mathbb{N}$ are the numbers of indices $i_{1}$ and $j_{1}$. Pixels outside this minimum distances have to be excluded from further processing. The lower value of $x_{\text {mindist }}$ and $y_{\text {mindist }}$ is the absolute minimum of a point $\left(x_{i_{1}, \text { start }}, y_{j_{1} \text {,start }}\right)$ to one of the edges of the CCD image and can be used as maximal allowed radius $r_{\max }$ for the circles defined beneath.

From $\left(x_{i_{1}, \text { start }}, y_{j_{1} \text {,start }}\right)$ as center $(=0)$ to $r_{\max }$ a number of subrings $n_{\text {subr }}$ with a variable segment width $\Delta r$ are created $(\Delta r=\Delta x=\Delta y=10 \mu \mathrm{m}$ is a good choice, since every pixel is captured and the segments are relatively broad, so many pixels are taken for averaging in the following):

$n_{\text {subr }}=r_{\max } / \Delta r$, i.e. $r_{i}=i \Delta r$ for $i=0, \ldots, n_{\text {subr }}$ is the $i$-th radii subdivision. Now find the maximum

$$
\max _{i=0, \ldots, n_{\mathrm{subr}}-1}\left(\frac{1}{N_{i}} n_{\mathrm{C}}\left(r_{i} \leq r<r_{i+1}\right)\right)
$$

for

$$
r_{i}=\sqrt{\left(x_{i}-x_{i_{1}, \text { start }}\right)^{2}+\left(y_{j}-y_{j_{1}, \text { start }}\right)^{2}},
$$

which sums up all pixel values $n_{\mathrm{C}}$ fulfilling the condition $r_{i} \leq r<r_{i+1}$ and divides them by the number of such pixels $N_{i}$ for every $i$. The radius $r_{i}$ with ring segment of maximum value is saved. This procedure is repeated for all the $\left(x_{i_{1} \text {,start }}, y_{j_{1}, \text { start }}\right)$ and finally the pixel $\left(x_{1, \text { start }}, y_{1, \text { start }}\right)$ with the highest average value for a ring segment of the innermost ring, which certainly has the peak average photoelectron values (see Fig. 4 (e) in 1D and Fig. 6 in 2D), is taken as best center.

Exactly the same process can be applied to the subpixelarea of $\left(x_{1, \text { start }}, y_{1, \text { start }}\right)$, by taking subintervals $\Delta x^{\prime}=\Delta y^{\prime}=$ $2 \mu \mathrm{m}$ of $\Delta x=\Delta y=10 \mu \mathrm{m}$ for example, thus yielding a $m_{2} \times n_{2}=5 \times 5$ matrix of squares $\left(m_{2}, n_{2} \in \mathbb{N}\right)$, and a best ring center $\left(x_{2, \text { start }}, y_{2 \text {,start }}\right)$ from all subpixels $\left(x_{i_{2}, \text { start }}\right.$, $y_{j_{2}, \text { start }}$ ) inside of them (right part of Fig. 9). The ring segment width stays constant, $\Delta r^{\prime}=\Delta r$. This way the center 


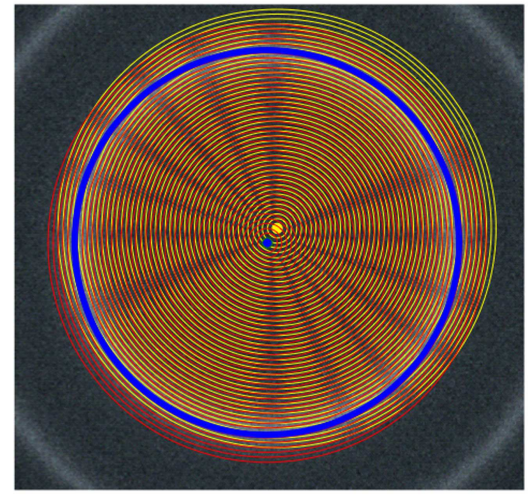

Fig. 10 Ring segments around center points: here the blue center (with the red rings around it) delivers the ring segment with a higher maximum value of mean photoelectrons (thick blue ring) than the yellow center (with the yellow rings) and is therefore chosen as best suited center.

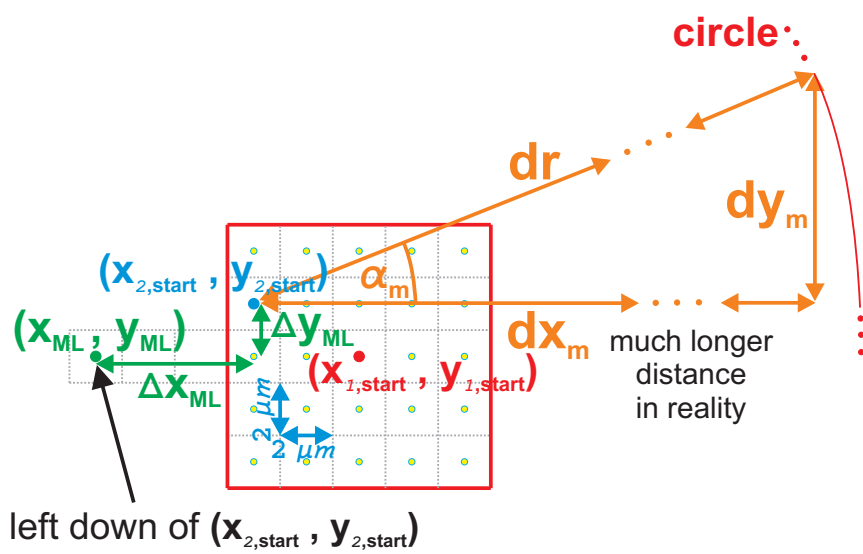

Fig. 11 Evaluation of radii via the Midpoint Line method: from the determined subpixel-center $\left(x_{2, \text { start }}, y_{2, \text { start }}\right)$ lines $d r$ of varying angles $\alpha_{m}$ (and therefore varying lengths $d x_{m}$ and $d y_{m}$ ) are subdivided into intervals of equal length, and the cuts with the $2 \mathrm{D}$ pixel values give the corresponding photoelectron numbers. An offset $\left(x_{\mathrm{ML}}, y_{\mathrm{ML}}\right)$ has to be considered.

of mass of each figure can be evaluated with a low bias and standard deviation, although the noise (see Figs. 6 (c),(d)) is quite high and it creates errors in finding the average maximum ring. Center evaluation is only possible for fringe patterns without peaks in the center (like in Fig. 6 (a)), but not for images like in Fig. 6 (b). The process described is quite time-consuming.

There may be cases, where the search with the $\left(x_{i_{1} \text {,start }}\right.$, $\left.y_{j_{1}, \text { start }}\right)$ yields a wrong value and then the search with the $\left(x_{i_{2}, \text { start }}, y_{j_{2}, \text { start }}\right)$ is worthless. Figure 10 summarizes the center evaluation procedure. In this case the yellow center point does not yield the maximum average in its radial ring segments, but for the blue center the thick blue ring is the segment with the highest mean value, and therefore the blue point finally is chosen as center for the following calculations.

\subsection{Evaluation of radii via Midpoint Line method}

The center $\left(x_{2, \text { start }}, y_{2, \text { start }}\right)$ serves as a starting point for the computation methods. The first one is a variant of Bresenham's midpoint line (ML) algorithm [74] extended to radial lines for every angle between $0^{\circ}$ and $360^{\circ}$ (i.e. $[0,2 \pi$ ) in radians) of circular environment, that is $1 \mathrm{D}$ cuts through the $2 \mathrm{D}$ rings. This implies a center offset consideration between the determined subpixel center $\left(x_{2, \text { start }}, y_{2, \text { start }}\right)$ and the center of the next whole pixel $\left(x_{\mathrm{ML}}, y_{\mathrm{ML}}\right)$ left below $\left(x_{2, \text { start }}, y_{2, \text { start }}\right)$, see Fig. 11. This approach is done to know the orientation and have the first half line, starting in $\left(x_{2}\right.$,start, $\left.y_{2, \text { start }}\right)$ with radial value 0 and angles $0^{\circ} \leq \alpha \leq 45^{\circ}$ to the right upwards related to the $x$-axis. The side lengths of each pixel are normalized (their lengths are 1 ). This way also the offset $\left(\Delta x_{\mathrm{ML}}, \Delta y_{\mathrm{ML}}\right)$ from $\left(x_{\mathrm{ML}}, y_{\mathrm{ML}}\right)$ to $\left(x_{2, \text { start }}, y_{2, \text { start }}\right)$ is expressed in this normalized lengths. A difference of one pixel on the $x$ - or $y$-axis is an index increment or decrement of 1 for indices $i$ and $j$ of the axes, respectively.

A circle, with an angular range of $360^{\circ}$, is divided into eight segments of $45^{\circ}$ each (because the following equations are only valid in this sector), i.e. $\alpha_{\min }=0^{\circ}, \alpha_{\max }=45^{\circ}$ are the minimum and maximum angles of each segment, and $\alpha_{\text {steps }}$ is the number of subdivisions over $360^{\circ}$. Therefore the angular resolution is $\alpha_{\text {res }}=8 \times\left(\alpha_{\max }-\alpha_{\min }\right) /\left(\alpha_{\text {steps }}\right)$, with $\alpha_{\text {steps }}=1440$ and $\alpha_{\text {res }}=0.25^{\circ}$ chosen here.

The $d x$ and $d y$ values vary with an integer $m$ for angles $\alpha_{m}$, and are therefore called $d x_{m}$ and $d y_{m}$ (see Fig. 11). With $d r^{2}=\left(d x_{m}\right)^{2}+\left(d y_{m}\right)^{2}$ and $d r=r_{\max }$ as constant maximum radius we get $d x_{m}=\cos \left(\alpha_{m}\right) d r$ and $d y_{m}=$ $\sqrt{d r^{2}-\left(d x_{m}\right)^{2}}$ for $0 \leq m<\alpha_{\max }$. A decision variable $d_{m}=2 d y_{m}-d x_{m}$ is needed, as well as increments for moving to the east (right), $i n_{\mathrm{E}, m}=2 d y_{m}$, and increments used for moving to the north-east (upwards-right), $i n_{\mathrm{NE}, m}=$ $2\left(d y_{m}-d x_{m}\right)$.

$\left(x_{2, \text { start }}, y_{2, \text { start }}\right)$ is the starting point and $i_{\mathrm{ML}}, j_{\mathrm{ML}}$ are the indices of the pixel center to its left downwards. As long as

$$
\sqrt{\left(d x_{m}\right)^{2}+\left(d y_{m}\right)^{2}} \leq d r=r_{\max }
$$

the following steps are executed: if $d_{m} \leq 0$, then set

$$
\begin{aligned}
d_{m}: & =d_{m}+i n_{\mathrm{E}, m}, \\
x_{m} & :=x_{m}+\left(d x_{m} / d r\right), \\
i & =i_{\mathrm{ML}}+\left\lfloor 0.5+x_{m}\right\rfloor .
\end{aligned}
$$

with $\lfloor\cdot\rfloor$ the largest integer that is less than or equal to the number. Else if $d_{m}>0$, then set

$$
\begin{aligned}
d_{m} & :=d_{m}+i n_{\mathrm{NE}, m}, \\
x_{m} & :=x_{m}+\left(d x_{m} / d r\right), \\
i & =i_{\mathrm{ML}}+\left\lfloor 0.5+x_{m}\right\rfloor, \\
y_{m} & :=y_{m}+\left(d x_{m} / d r\right), \\
j & =j_{\mathrm{ML}}+\left\lfloor 0.5+y_{m}\right\rfloor .
\end{aligned}
$$

Finally an integer $k$ counts the number of repetitions of steps (30) and (31), as long as (29) is valid, and $k$ defines the multiples of the normalized side lengths and this way the distance 
from the center. Note that this solely describes the procedure for angles $0^{\circ} \leq \alpha_{m}<45^{\circ}$. The formulas in (30) and (31) and before need only be slightly adapted for the other $7 \mathrm{sec}-$ tors covering $45^{\circ}$ each. For example, $d x_{m}=-\cos \left(\alpha_{m}\right) d r$, or $d_{m}=2 d x_{m}-d y_{m}$, or $y_{m}:=y_{m}-\left(d x_{m} / d r\right)$ and $j=j_{\mathrm{ML}}-\left\lfloor 0.5+y_{m}\right\rfloor$, and so on, are possible changes, that have to be combined due to geometrical and symmetrical considerations for all sectors.

What we finally get from each line is a function of photoelectron values $n_{\mathrm{C}}$ depending on radius values (pixels) from the center (see the red function in Fig. 12). A difficulty is the noise of such a single line, as shown in Fig. 12 for an angle of $\alpha=269^{\circ}$. Although a Savitzky-Golay filter (SG) $[67,75,76]$ provides a good estimate and smooths noisy data of such non-equidistant fringes of different widths, it is not enough to just fit the single $1 \mathrm{D}$ lines (cuts) by this filter. Indeed fitting 360 noisy cuts in steps of $1^{\circ}$ with SG at the conditions of Table 1 with the higher number of $2.4 \times 10^{7}$ photons (backscattered from $56 \mathrm{~m}$ distance) delivers big random calculation errors. Taking 37 smoothing filter points left and right $\left(n_{L}=n_{R}=37\right)$ and a polynomial degree of 4 , and comparing each fit to the expected $v_{\mathrm{LOS}}$-value yields a mean velocity calculation error (bias) of more than $50 \frac{\mathrm{m}}{\mathrm{s}}$ (see Secs. $5.4,5.5$ and 6 for further details on fitting). Disadvantages of $\mathrm{SG}$ are the loss of points on the edge of the domain (so only 37 points left and right could be used to get the second ring and its peak still fitted) and that $n_{L}$ and $n_{R}$ will not be suited for every ring width (e.g. $n_{L}=n_{R}=37$ may be useful for broader rings, but bad for narrow ones, and vice versa). Fitting with a polynomial periodic function is even more unsuitable than SG, since they will not keep the positions of the peaks, especially if they are nonequidistant.

Different to that, the power of our algorithms lies in the averaging procedure for a great number of such lines, taking pixel numbers and their corresponding electron numbers. The radially calculated photoelectron values $n_{\mathrm{C}}$ must be summed up for positions of equal distance to the center $\left(x_{2, \text { start }}, y_{2, \text { start }}\right)$ and divided by $\alpha_{\text {steps }}$, yielding averaged real numbers for the mean photoelectrons. The positions in units of pixels are multiplied by the pixelsize of $10 \mu \mathrm{m}$ and the result is shown in Fig. 13 (a) for the two innermost rings for three different wavelengths (i.e. CCD images), showing averaged photoelectron numbers $n_{\mathrm{C}, \text { mean }, i}$ in dependence on the radii values $r_{\text {mean }, i}$ for $i=0, \ldots, n_{\text {subr }}-1$. An angular resolution of $0.25^{\circ}$ of a circle is necessary (as the results in Table 2 will show) for good results, that reduce the noise drastically and visible, see Fig. 13 (a). At steps of $1^{\circ}$ some pixels far away from the center may not be hit by the lines and this way not be incorporated in the calculations.

A drawback of this method is the choice of the same value sequentially on a single line for different $k$, because the step size is only $\Delta x=\Delta y=1 \geq \Delta r$. At an angle of $\pi / 4$ for example, the extreme case of $\Delta r=\cos (\pi / 4)=0.707<1$ is the reason for having a low value $d x_{m}$, and therefore the same electron value is chosen for two different radial and equidistant positions, which distorts the results. The same is true for angles of $\alpha_{m}=(\pi / 4)+k^{\prime}(\pi / 2), k^{\prime} \in \mathbb{Z}$. The opposite case

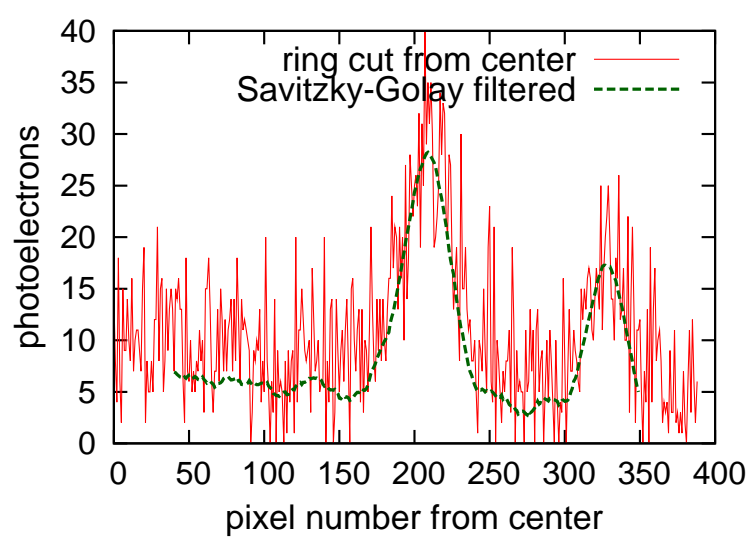

Fig. 12 Evaluation: single cut from center to the edge (calculated by ML-method) at an angle of $269^{\circ}$ and filtered with Savitzky-Golay.

for $\alpha_{m}=k^{\prime}(\pi / 2), k^{\prime} \in \mathbb{Z}$ is optimal, since then the cuts through the $2 \mathrm{D}$ rings lie on the $x$ - or $y$-axes and according to $\Delta x=\Delta y=1=\Delta r$ no electron values are taken two times.

\subsection{Evaluation of radii via Circular Averaging method}

The second method, called circular averaging method (CA), works like the center evaluation procedure from Sec. 5.1. Ring segments of the formerly used width $\Delta r$ and the calculated best ring center $\left(x_{2, \text { start }}, y_{2}\right.$,start $)$ are taken, and the sum of their photoelectron numbers are averaged:

$$
\begin{aligned}
n_{\mathrm{C}, \text { mean }, i} & =\frac{1}{N_{i}} n_{\mathrm{C}}\left(r_{i} \leq r<r_{i+1}\right) \text { at radii positions } \\
r_{\text {mean }, i} & =0.5\left(r_{i+1}-r_{i}\right) \text { for } i=0, \ldots, n_{\mathrm{subr}}-1 \\
\text { with } r_{i} & =i \Delta r \text { and } 0 \leq r \leq r_{\max } .
\end{aligned}
$$

Figure 13 (b) shows $n_{\mathrm{C}, \text { mean }, i}$ in dependence on $r_{\text {mean }, i}$ calculated with the CA method for the same three wavelengths as does Fig. 13 (a) for the ML method. No differences are observable on a scale of Fig. 13 due to their tinyness, although they indeed exist.

\subsection{Fitting procedure}

Although the fringe patterns in Fig. 13 look quite smooth and the radii positions of the peaks might serve as good radius (wavelength) values, improvement is necessary and possible by a fitting procedure, that was also applied to the SG filtered lines mentioned afore. A threshold is set to cut out the peaks of the rings (see Fig. 14 for the innermost rings averaged with the ML method) and then separately fitting them nonlinearly by use of the Levenberg-Marquart method [67] to a polynomial $p(r)=a r^{2}+b r+c$ of degree two with $a, b, c \in \mathbb{R}$. The radius is determined from the maximum position as $r=-b /(2 a)$. To have enough points to fit (here it is 30 to 50 points approximately), the rings should be not too sharp (lower finesse of the FPI or strong molecular broadening). 


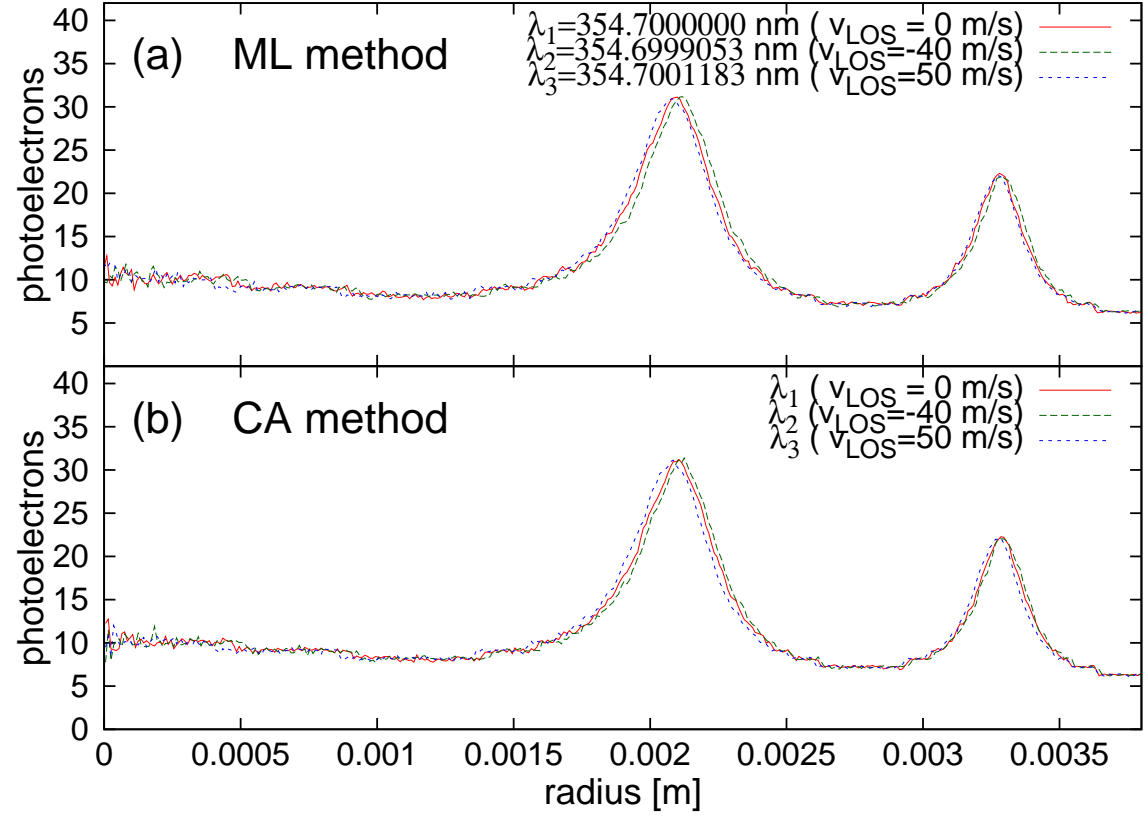

Fig. 13 Evaluation: fringes averaged for three wavelengths by (a) ML method; (b) CA method.

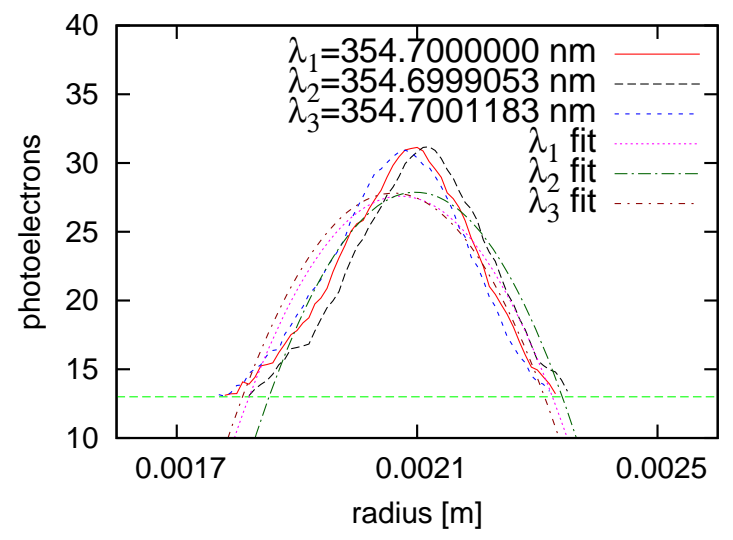

Fig. 14 Evaluation: fitting procedure with Levenberg-Marquardt nonlinear least squares fitting for exact radii determination for rings averaged with ML at three different wavelengths; a threshold excludes values below.

The signal pattern may also be fit to Gaussian- or Airyshaped patterns, which may reduce the deviation. But this would require a number of physical properties of the optics and the environment known or measured very precisely, especially since the fringes broaden or narrow with changing flight level. Our approach will completely do without them after calibration and under the assumption of stable wavelength and optical alignment.

\subsection{System calibration}

For system calibration a good wavemeter for absolute wavelength measurements should be sufficient (a precision of $v_{\mathrm{LOS}}$ of $1 \frac{\mathrm{m}}{\mathrm{s}}$ requires a wavelength accuracy of about $2.4 \mathrm{fm}$ ). Fre-

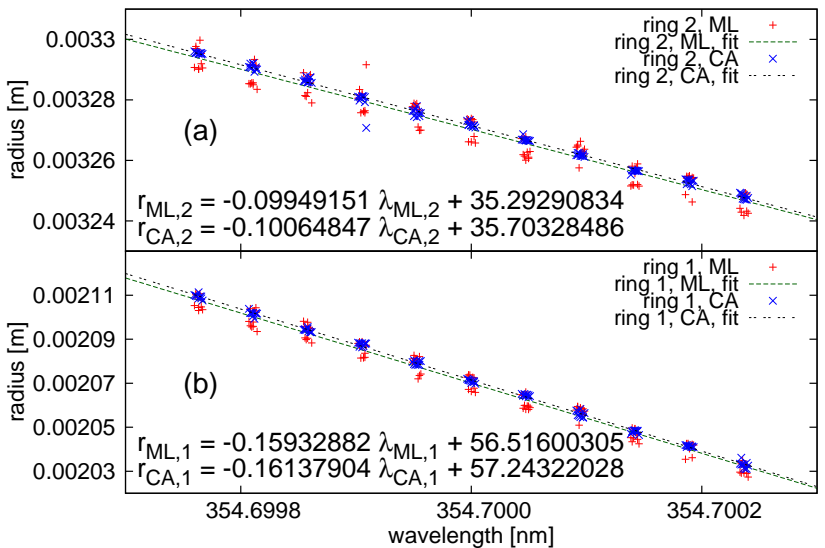

Fig. 15 Calibration lines for the two inner rings ( (a) for the second ring, (b) for the innermost ring; radii calculated via the ML and CA methods) at tuned wavelengths: the equations include the calculated relation between the wavelengths and the radii.

quency combs (FCs) have revolutionized the way and precision the wavelength of light can be measured [77,78] and are a highly suitable source for wavelength determination of a cw-laser. For calibration a low-power, frequency-tripled, slightly tunable Nd:YAG cw-laser is proposed here. The nearly monochromatic laser light will generate narrower and steeper rings and peaks on the $\mathrm{CCD}$, thus having less points to fit, but with less noise, too. The CCD's resolution is the limiting factor for radii determination and thus calibration. The tunable laser emits a beam, that is split into two parts: one for the FPI in the mode it will be operating later under fixed plate distance, with the CCD at the end of the light propagation, and the other for the wavemeter or FC. The CCD diagrams radii 
are determined the way described before via the CA or ML methods, and the wavemeter or FC measures the corresponding wavelengths.

Alternatively for calibration of the radii to the corresponding wavelengths, a laser pulse or cw laser radiation is emitted towards a disc with a certain rotational velocity $[15,25$, 28]. This moving disc replaces the particles of the atmosphere (or the tunable laser) and the backscattered photons receive a wavelength shift according to the Doppler shift formula [48]

$$
\Delta \lambda=-2 \lambda_{1} v_{\mathrm{LOS}} c^{-1}
$$

at an angle of $180^{\circ}$, where $\lambda_{1}=354.7 \mathrm{~nm}$ (also in Figs. 13 and 14) is the reference wavelength and $\Delta \lambda$ the wavelength shift.

We simulate ten measurements for each velocity $v_{\mathrm{LOS}}$ from $-100 \frac{\mathrm{m}}{\mathrm{s}}$ to $+100 \frac{\mathrm{m}}{\mathrm{s}}$ in steps of $20 \frac{\mathrm{m}}{\mathrm{s}}$ equal to wavelengths from $354.7002366 \mathrm{~nm}$ to $354.6997634 \mathrm{~nm}$. As just mentioned, a LOS-component of $v_{\mathrm{LOS}}=1 \frac{\mathrm{m}}{\mathrm{s}}$ corresponds to a wavelength shift of only $\Delta \lambda=2.4 \mathrm{fm}$ at $\lambda_{1}=354.7 \mathrm{~nm}$. A standard deviation of $1 \frac{\mathrm{m}}{\mathrm{s}}$ for the wavelength was assumed here. The FSR at $\lambda_{1}=354.7 \mathrm{~nm}$ and $d=6.5 \mathrm{~mm}$ is $0.01 \mathrm{~nm}$, i.e. sufficient to get no inseparable overlap. The beam is weakened to 100 million photons that reach the CCD with a pixel size of $10 \times 10 \mu \mathrm{m}^{2}$ and $961 \times 781$ pixels, so saturation effects are minimized. Speckle noise is taken into account with $\bar{s}=8.5$ [70] (only during calibration). Calibration values and the resulting lines due to linear relationship as well as the formulas for conversion from $r$ to $\lambda$, calculated with the ML and CA methods, are shown in Figs. 15 (a) and (b) for the second and the innermost ring, respectively. It was assumed that the ring centers only move within the same pixel during calibration. An error of more than one pixel for the radius from noisy rings will lead to a huge error when calculating $\lambda$ with the conversion formulas.

\section{Assessment of the calculation results}

For determination of $v_{\text {LOS }}$ and thus wind estimation [19,79, 80], the wavelengths corresponding to the evaluated radii will be calculated out of the equations mentioned in Fig. 15 (a) and (b), and are compared to the set values of the simulated noisy FPI CCD images. This way the usefulness of the algorithms can be tested, see Table 2 for a value of $\lambda=354.7 \mathrm{~nm}$, that is $v_{\mathrm{LOS}}=0 \frac{\mathrm{m}}{\mathrm{s}}$. The $\lambda$ and $v_{\mathrm{LOS}}$ are written in the form $a \pm b$, where $a$ is the measurement or calculation bias and $b$ its standard deviation. The standard deviation for the ML algorithm is much too high, so the CA method should be chosen. This means the results are calculated more stable with CA, see the lower standard deviations around the mean of $7 \frac{\mathrm{m}}{\mathrm{s}}$ after 20 evaluated diagrams compared to ML calculations in Table 2 . The deviation to $v_{\mathrm{LOS}}=0 \frac{\mathrm{m}}{\mathrm{s}}$ can be strongly reduced by more photons at equal CCD resolution and pixel size and by more measured diagrams. However, the results are relatively stable (low standard deviations) for the CA method after only 20 CCD images, which is essential at flight speed.
Note that these results are valid for the case of moving centers, where the center evaluation has to be applied. If the center is stationary and exactly known, $v_{\mathrm{LOS}}$ can be determined better than $2 \frac{\mathrm{m}}{\mathrm{s}}$ for the innermost ring, averaging the radii calculated of 20 CCD images at $\lambda=354.7 \mathrm{~nm}$ with CA for two photon numbers $n_{1}$ and $n_{2}$, as shown in Table 3 . The error for the second ring is huge; this may be due to the lower number of points for the Levenberg-Marquardt fit.

Doubling the pixel sidelength to $20 \mu \mathrm{m}$ and reducing the CCD resolution to $481 \times 391$ pixels will greatly decrease the calculation time, but the precision will suffer immensely. The best value achievable after evaluation of 20 CCD images with 24 million photons distributed on each, was more than $30 \frac{\mathrm{m}}{\mathrm{s}}$ away from the expected $0 \frac{\mathrm{m}}{\mathrm{s}}$ (not shown). The benefit of about four times the number of photoelectrons for each pixel than for $961 \times 781 \mathrm{CCD}$ resolution is not enough to outweigh the drawback of having less pixels to fit with LevenbergMarquardt.

\section{Conclusion and outlook}

The in-depth simulation of realistic noisy 2D FPI rings and two universally applicable methods to determine the wavelength of low intensity, backscattered radiation at an altitude of $8.5 \mathrm{~km}$ from them were illustrated in detail. The CA method proved to be the more reliable and precise one. A main advantage of FI compared to others like the edge technique is its ability to not depend on the mixing ratio of aerosol and molecular scattering, and that once calibration for a CCD of certain resolution was done, it can be used for every altitude level in the atmosphere. Aerosols and molecules need not be separated from each other, allowing a stronger throughput of received light to the CCD. FI techniques are less limited in the range of measureable wind speeds than the edge techniques. Furthermore FI has linear increments in wavelength (frequency) in contrast to the edge methods, and does not require knowledge of the atmospheric temperature for wind retrieval. The wavelength can not only be determined relatively, but in absolute values, and the shape and steepness of the fringes may vary. Drawbacks of our approach are the required high precision and, as a consequence, a huge period of time to calculate the results of the ring diagrams. However, simplification is possible and computers will become faster. Diffraction-limiting circular instruments like telescopes, diaphragms or lenses have to be tested for fulfilling the Rayleigh criterion, and the system's etendue (product of aperture and divergence) may be a crucial factor.

Concerning the laser frequency it could be sufficient to stabilize the source by using a wavemeter, since the FSR offers a certain part of tolerance (at least $\pm 0.002 \mathrm{~nm}$ around the center wavelength reasonably, see Fig. 5). We assumed optimal operation conditions of the system, i.e. mechanically stable optical device positions and manufacturing without defects as well as stable temperature conditions to exclude changes of the refractive index inside the optical path. These difficulties have been analysed in detail $[23,24,70]$. Note that the 
Table 2 Average results after 20 calculated 2D CCD noisy ring diagrams at $v_{\mathrm{LOS}}=0 \frac{\mathrm{m}}{\mathrm{s}}$ (equivalent to $\lambda=354.7 \mathrm{~nm}$ ) for $n_{1}=1.3 \cdot 10^{7}$ photons (refering to a measurement distance of $76 \mathrm{~m}$ ) and $n_{2}=2.4 \cdot 10^{7}$ photons (refering to a measurement distance of $56 \mathrm{~m}$ ) without known center (center has to be calculated).

\begin{tabular}{ccccc}
\hline calc. method & ML, ring 1 & ML, ring 2 & CA, ring 1 & CA, ring 2 \\
\hline radius $[\mathrm{m}], n_{1}$ & 0.00207632 & 0.003264339 & 0.0020804445 & 0.0032751615 \\
radius $[\mathrm{m}], n_{2}$ & 0.00207190 & 0.003271150 & 0.0020743290 & 0.0032732625 \\
$\lambda[\mathrm{nm}], n_{1}$ & $354.699961 \pm 0.000042$ & $354.700060 \pm 0.000327$ & $354.699944 \pm 0.000006$ & $354.699963 \pm 0.000007$ \\
$\lambda[\mathrm{nm}], n_{2}$ & $354.699988 \pm 0.000053$ & $354.699991 \pm 0.000093$ & $354.699982 \pm 0.000006$ & $354.699982 \pm 0.000007$ \\
$v_{\mathrm{LOS}}\left[\frac{\mathrm{m}}{\mathrm{s}}\right], n_{1}$ & $-16.67 \pm 17.87$ & $25.13 \pm 138.33$ & $-23.58 \pm 2.59$ & $-15.47 \pm 3.06$ \\
$v_{\mathrm{LOS}}\left[\frac{\mathrm{m}}{\mathrm{s}}\right], n_{2}$ & $-4.95 \pm 23.39$ & $-3.80 \pm 39.11$ & $-7.57 \pm 2.48$ & $-7.49 \pm 2.89$ \\
\hline
\end{tabular}

specifications of a final measurement system need careful consideration, that goes beyond the scope of this article. The parameter values specified here are estimated on a best effort basis and can be different from real instrumental design. The essential part of this work was to describe the methods to determine the ring radii. Although the analysis is restricted to one special case at $8.5 \mathrm{~km}$ height and to two measurement distances, this approach will be applicable for every atmospheric backscatter condition, and its only limitations are the readout-speed from the $\mathrm{CCD}$, the $\mathrm{CCD}$ resolution, the data processing time and the measurement distance. The methods could also be useful for high-resolution laser spectroscopy.

Nonetheless a multitude of further investigations concerning the CCD's domain resolution and pixel sizing, and the FPI's plate dimensions and air-gap spacings (air-gap étalon, aperture in $\mathrm{cm}$ ) are possible. Airy- or Gaussian-fits could be applied instead of Levenberg-Marquardt. Tests with real optical components, that also have distortions like plate defects and detector nonlinearities [21], will show the feasibility of the proposed approach.

Table 3 Average results after 20 calculated 2D CCD noisy ring diagrams at $v_{\mathrm{LOS}}=0 \frac{\mathrm{m}}{\mathrm{s}}$ (equivalent to $\lambda=354.7 \mathrm{~nm}$ ) for $n_{1}=1.3 \cdot 10^{7}$ and $n_{2}=2.4 \cdot 10^{7}$ photons with exactly known center.

\begin{tabular}{ccc}
\hline calc. method & CA, ring 1 & CA, ring 2 \\
\hline$v_{\text {LOS }}\left[\frac{\mathrm{m}}{\mathrm{s}}\right], n_{1}$ & $1.06 \pm 3.17$ & $23.55 \pm 4.71$ \\
$v_{\text {LOS }}\left[\frac{\mathrm{m}}{\mathrm{s}}\right], n_{2}$ & $1.56 \pm 1.74$ & $19.16 \pm 2.89$ \\
\hline
\end{tabular}

Acknowledgements This work was conducted in the framework of the DLR-project "Wetter \& Fliegen". The first author wants to thank $O$. Reitebuch for internal reviewing this article and for helpful comments on its improvement, as well as all members of IPA's lidar group for fruitful discussions and helpful advice. He is also grateful to F. Holzäpfel for reviewing a shorter prior version of this article.

\section{References}

1. C. Werner, Doppler Wind Lidar, in Lidar: Range-Resolved Optical Remote Sensing of the Atmosphere, ed. by C. Weitkamp, in Springer Series in Optical Sciences, Springer, New York, 2005, pp. 325-354.
2. R. Frehlich, S.M. Hannon, S.W. Henderson, J. Atmos. Ocean. Technol. 11, 1517 (1994).

3. S. Rahm, Opt. Lett. 26, 319 (2001).

4. O. Reitebuch, C. Werner, I. Leike, P. Delville, P.H. Flamant, A. Cress, D. Engelbart, J. Atmos. Ocean. Technol. 18, 1331 (2001).

5. H. Inokuchi, H. Tanaka, T. Ando, Proceedings of the 26th international congress of the aeronautical sciences, 1-8 (2008).

6. A. Dolfi-Bouteyre, G. Canat, M. Valla, B. Augère, C. Besson, D. Goular, L. Lombard, J.-P. Cariou, A. Durecu, D. Fleury, L. Bricteux, S. Brousmiche, S. Lugan, B. Macq, IEEE J. Sel. Top. Quantum Electron. 15, 441 (2009).

7. M.L. Chanin, A. Garnier, A. Hauchecorne, J. Porteneuve, Geophys. Res. Lett. 16, 1273 (1989).

8. A. Garnier, M.-L. Chanin, Appl. Phys. B 55, 35 (1992).

9. C.L. Korb, B.M. Gentry, C.Y. Weng, Appl. Opt. 31, 4202 (1992).

10. J.A. McKay, Appl. Opt. 37, 6480 (1998).

11. C.L. Korb, B.M. Gentry, S.X. Li, C. Flesia, Appl. Opt. 37, 3097 (1998).

12. C. Souprayen, A. Garnier, A. Hertzog, Appl. Opt. 38, 2422 (1999).

13. B.M. Gentry, H. Chen, S.X. Li, Opt. Lett. 25, 1231 (2000).

14. O. Reitebuch, C. Lemmerz, E. Nagel, U. Paffrath, Y. Durand, M. Endemann, F. Fabre, M. Chaloupy, J. Atmos. Ocean. Technol. 26, 2501 (2009).

15. Z.S. Liu, W.B. Shen, T.L. Zhang, J.W. Hair, C.Y. She, Appl. Phys. B 64, 561 (1997).

16. J.N. Forkey, W.R. Lempert, R.B. Miles, Appl. Opt. 36, 6729 (1997).

17. Z.S. Liu, B.Y. Liu, Z.G. Li, Z.A. Yan, S.H. Wu, Z.B. Sun, Appl. Phys. B 88, 327 (2007).

18. D. Rees, I.S. McDermid, Appl. Opt. 29, 4133 (1990).

19. C.A. Tepley, S.I. Sargoytchev, R. Rojas, IEEE Trans. Geosci. Remote Sensing 31, 36 (1993).

20. K.W. Fischer, V.J. Abreu, W.R. Skinner, J.E. Barnes, M.J. McGill, T.D. Irgang, Opt. Eng. 34, 499 (1995).

21. M.J. McGill, W.R. Skinner, T.D. Irgang, Appl. Opt. 36, 1928 (1997).

22. N. Cézard, A. Dolfi-Bouteyre, J.-P. Huignard, P. H. Flamant, Appl. Opt. 48, 2321 (2009).

23. J.A. McKay, Appl. Opt. 37, 6487 (1998).

24. M.J. McGill, J.D. Spinhirne, Opt. Eng. 37, 2675 (1998).

25. N.P. Schmitt, W. Rehm, T. Pistner, P. Zeller, H. Diehl, P. Navé, Aerosp. Sci. Technol. 11, 546 (2007).

26. G. Jenaro Rabadan, N. P. Schmitt, T. Pistner, W. Rehm, Journal of Aircraft 47, 392 (2010).

27. S.C. Snyder, L.D. Reynolds, G.D. Lassahn, J.R. Fincke, C.B. Shaw Jr., R.J. Kearney, Phys. Rev. E 47, 1996 (1993).

28. R.L. McKenzie, Appl. Opt. 35, 948 (1996). 
29. R.G. Seasholtz, A.E. Buggele, M.F.Reeder, Opt. Lasers Eng. 27, 543 (1997).

30. M.M. Clem, A.F. Mielke-Fagan, K.A. Elam, "Study of FabryPerot Etalon Stability and Tuning for Spectroscopic Rayleigh Scattering," 48th AIAA Aerospace Sciences Meeting Including the New Horizons Forum and Aerospace Exposition, Orlando, Florida, Jan. 4-7, 2010, AIAA 2010-855.

31. T.L. Killeen, B.C. Kennedy, P.B. Hays, D.A. Symanow, D.H. Ceckowski, Appl. Opt. 22, 3503 (1983).

32. P.B. Hays, Appl. Opt. 29, 1482 (1990).

33. J. Wu, J. Wang, P.B. Hays, Appl. Opt. 33, 7823 (1994).

34. M.J. McGill, M. Marzouk, V.S. Scott, J.D. Spinhirne, Opt. Eng. 36, 2171 (1997).

35. J.M. Vaughan, The Fabry-Perot Interferometer (Adam Hilger, Bristol, 1989).

36. G. Hernandez, Fabry-Perot interferometers (Cambridge University Press, 1986).

37. K.W. Meissner, J. Opt. Soc. Am. 31, 405 (1941).

38. N. Barakat, M. Medhat, Optica Acta 33, 939 (1986).

39. T.J. Scholl, S.J. Rehse, R.A. Holt, S.D. Rosner, Rev. Sci. Instrum. 75, 3318 (2004).

40. M. O’Hora, B. Bowe, V. Toal, J. Opt. A: Pure Appl. Opt. 7, 364 (2005).

41. T. Schröder, C. Lemmerz, O. Reitebuch, M. Wirth, C. Wührer, R. Treichel, Appl. Phys. B 87, 437 (2007).

42. V.A. Banakh, I.N. Smalikho, F. Köpp, C. Werner, Appl. Opt. 34, 2055 (1995).

43. S.R. Pal, A.I. Carswell, Appl. Opt. 15, 1990 (1976).

44. L.R. Bissonnette, P. Bruscaglioni, A. Ismaelli, G. Zaccanti, A. Cohen, Y. Benayahu, M. Kleiman, S. Egert, C. Flesia, P. Schwendimann, A.V. Starkov, M. Noormohammadian, U.G. Oppel, D.M. Winker, E.P. Zege, I.L. Katsev, I.N. Polonsky, Appl. Phys. B 60, 355 (1995).

45. L.I. Chaikovskaya, E.P. Zege, I.L. Katsev, M. Hirschberger, U.G. Oppel, Appl. Opt. 48, 623 (2009).

46. D. Pierrottet, F. Amzajerdian, L. Petway, B. Barnes, G. Lockard, Proc. SPIE 7323, 732311 (2009).

47. N. Lindlein, G. Leuchs, Geometrical Optics, Chap. 2.4 Ray Tracing, in Springer Handbook of Lasers and Optics, ed. by F. Träger, Springer, New York, 2007, pp. 61-67.

48. W. Demtröder, Laser Spectroscopy, Vol. 1: Basic Principles (Springer, Berlin Heidelberg, 4th ed. 2008).

49. A. Ansmann, U. Wandinger, O. Le Rille, D. Lajas, A.G. Straume, Appl. Opt. 46, 6606 (2007).

50. U. Paffrath, C. Lemmerz, O. Reitebuch, B. Witschas, I. Nikolaus, V. Freudenthaler, J. Atmos. Ocean. Technol. 26, 2516 (2009).

51. J.M. Vaughan, D.W. Brown, C. Nash, S.B. Alejandro, G.G. Koenig, J. Geophys. Res., 100 (D1), 1043 (1995).

52. J.M. Vaughan, N.J. Geddes, P.H. Flamant, C. Flesia, "Establishment of a backscatter coefficient and atmospheric database," ESA contract 12510/97/NL/RE, 110 p., (1998).

53. A.T. Young, Phys. Today 35, 42-48 (January 1982).

54. A.T. Young, G.W. Kattawar, Appl. Opt. 22, 3668 (1983).

55. C.-Y. She, Appl. Opt. 40 4875, (2001).

56. R.B. Miles, W.R. Lempert, J.N. Forkey, Meas. Sci. Technol. 12, R33-R51 (2001).

57. B.-Y. Liu, M. Esselborn, M. Wirth, A. Fix, D.-C. Bi, G. Ehret, Appl. Opt. 48, 5143 (2009).

58. B. Witschas, M. Vieitez, E.-J. van Duijn, O. Reitebuch, W. van de Water, W. Ubachs, Appl. Opt. 49, 4217 (2010).

59. R.T.H. Collis, P.B. Russell, "Lidar measurement of particles and gases by elastic backscattering and differential absorption," in
Laser Monitoring of the Atmosphere, ed. by E.D. Hinkley, Topics in Applied Physics, Vol. 14, Springer, Berlin, Heidelberg, 1976, pp. 71-151.

60. R.M. Measures, Laser Remote Sensing (Wiley, Florida, 1992).

61. G.J. Marseille, A. Stoffelen, Q. J. R. Meteorol. Soc. 129, 3079 (2003).

62. K.S.W. Champion, "Standard and reference atmospheres," in Handbook of geophysics and the space environment, ed. by A.S. Jursa, United States Air Force Geophysics Laboratory, 141 (1985)

63. G.A. Reider, photonik (Springer, Wien New York, 2nd ed. 2005).

64. http://www.pco.de/intensified-cameras/dicam-pro/, 2010.

65. K. Arisaka, Nucl. Instr. and Meth. A 442, 80 (2000).

66. J.E. Carranza, E. Gibb, B.W. Smith, D.W. Hahn, J.D. Winefordner, Appl. Opt. 42, 6016 (2003).

67. W.H. Press, S.A. Teukolsky, W.T. Vetterling, B.P. Flannery, Numerical recipes: the art of scientific computing (Cambridge, 3rd ed., 2007).

68. D. Dussault, P. Hoess, Proc. SPIE, 5563, 195 (2004).

69. Z. Liu, W. Hunt, M. Vaughan, C. Hostetler, M. McGill, K. Powell, D. Winker, Y. Hu, Appl. Opt. 45, 4437 (2006).

70. N. Cezard, A. Dolfi-Bouteyre, J.-P. Huignard, P. Flamant, Proc. of SPIE, 6750, 0801-0810 (2007).

71. G. Ehret, C. Kiemle, M. Wirth, A. Amediek, A. Fix, S. Houweling, Appl. Phys. B 90, 593 (2008).

72. B. Mayer, A. Kylling, Atmos. Chem. Phys. Discuss., 5, 1319 (2005).

73. C. Flesia, C.L. Korb, Appl. Opt. 38, 432 (1999).

74. J.E. Bresenham, IBM Systems Journal 4, 25 (1965).

75. A. Savitzky, M.L.E. Golay, Anal. Chem. 36, 1627 (1964).

76. H. Ziegler, Appl. Spectrosc. 35, 88 (1981).

77. S.A. Diddams, D.J. Jones, J. Ye, S.T. Cundiff, J.L. Hall, J.K. Ranka, R.S. Windeler, R. Holzwarth, Th. Udem, T.W. Hänsch, Phys. Rev. Lett. 84, 5102 (2000).

78. Th. Udem, R. Holzwarth, T.W. Hänsch, Nature 416, 233 (2002).

79. R. Frehlich, J. Atmos. Oceanic Technol. 18, 1628 (2001).

80. F. Andreucci, M.V. Arbolino, IEEE Geoscience \& remote sensing Symposium, 1995, Vol. 3, pp. 2319-2322. 